\title{
Radiation-Induced Condensational Growth and Cooling of Cloud-Sized Mist Droplets
}

\author{
M. Q. Brewster, ${ }^{a}$ X. Li, ${ }^{\mathrm{b}}$ K. K. Roman, ${ }^{\mathrm{a}, \mathrm{c}}$ E. O. MCNichols, ${ }^{\mathrm{a}, \mathrm{d}}$ AND M. J. RoOD ${ }^{\mathrm{b}}$ \\ ${ }^{a}$ Department of Mechanical Science and Engineering, University of Illinois at Urbana-Champaign, Urbana, Illinois; \\ ${ }^{\mathrm{b}}$ Department of Civil and Environmental Engineering, University of Illinois at Urbana-Champaign, Urbana, Illinois
}

(Manuscript received 21 October 2019, in final form 1 August 2020)

\begin{abstract}
A laboratory-experimental and theoretical-modeling investigation was conducted of isobaric, radiative cooling of cloud-like water mists to a remote heat sink, similar to what can happen at the tops of clouds. For mist initially at $20^{\circ} \mathrm{C}$ cooled by a radiative sink at $-20^{\circ} \mathrm{C}$, the mean $\left(D_{43}\right)$ mist droplet diameter grew from 5.5 to $8.4 \mu \mathrm{m}$ and the mist temperature decreased from $20^{\circ}$ to $3^{\circ} \mathrm{C}$ in just $80 \mathrm{~s}$. Modeling showed that conventional assumptions were able to predict the measured temperature decrease reasonably well but not droplet size changes, suggesting that bulk radiative cooling was being reasonably well modeled but not detailed, droplet-size-dependent behavior. In a theoretical analysis, Lewis-number near unity was exploited to obtain an analytic expression for quasi-steady supersaturation that agrees with what Davies reported in 1985 but is simpler and is a function of only droplet size distribution, surface tension, and solute parameters and not radiative transfer. A simpler expression for the corresponding time constant was also found that is a function of only the binary diffusion coefficient and $D_{31}$ moment of the droplet diameter distribution. The time constant was found to be in milliseconds and not seconds. Simply modifying quasi-steady supersaturation (i.e., applying droplet cooling effects uniformly to all droplet sizes) was shown not to be an acceptable substitute for including droplet-specific radiation terms in the droplet growth equation. These results confirm that radiative cooling at cloud top can have a significant effect on droplet size evolution and temperature change and provide data and analytical simplifications for use in further needed investigations of radiation modeling assumptions and parameterizations.
\end{abstract}

KEYWORDS: Cloud microphysics; Infrared radiation; Longwave radiation

\section{Introduction, background, motivation, and objectives}

The potential importance of the effect of thermal radiation on cloud droplet evolution and behavior has been known for well over a century. In 1877 Osborne Reynolds pointed out that, at cloud top, longwave (LW) thermal radiation between cloud droplets and a much colder, remote radiative heat sink such as outer space could induce a temperature difference between droplets and surrounding air that was of same order as that induced by diffusion (i.e., evaporative-condensative latent heat release and heat conduction), which is about onehundredth of a degree (Reynolds 1877; Fuchs 1959). Albeit an important observation, which has been referred to as the Reynolds effect by some, this effect was largely ignored for decades. In 1959, Fuchs (1959) included a droplet radiation term in the diffusional droplet growth equation; however, he considered radiation between droplets and surrounding air, not a cold, remote heat sink. In his brief discussion, the water droplet was considered to be a blackbody with a correction constant, which he deemed slightly smaller than unity but did not give an exact value. He (correctly) concluded that since the temperature difference between the droplet and the surrounding air is very small $\left(\sim 0.01^{\circ} \mathrm{C}\right)$, the radiative effect on droplet growth from surrounding vapor could be neglected.

\footnotetext{
${ }^{\mathrm{c}}$ Current affiliation: Department of Mechanical Engineering, State University of New York College of Technology at Canton, Canton, New York.

${ }^{\mathrm{d}}$ Current affiliation: NASA Glenn Research Center, Cleveland, Ohio.
}

Corresponding author: M. Q. Brewster, brewster@illinois.edu
In the 1970s Roach (1976) and Barkstrom (1978) rediscovered or revisited the idea that droplets residing at cloud top may experience a much larger radiative heat flux than droplets deep inside the cloud and studied theoretically the radiation effect on a top-of-cloud (or top-of-fog) droplet. Both studies showed that radiation can enhance droplet growth rate by as much as 20 times that with conduction alone. They also showed that larger droplets (on the order of tens of micrometers in diameter) could grow through radiative cooling even when surrounding air was subsaturated. The latter conclusion was later challenged (Davies 1985) on the basis that kinetic effects had not been included for the smallest droplets.

In the meantime, the scientific community had been puzzled by unexplained fast growth of raindrop embryos (droplets of hundreds of micrometers in diameter). Observations had shown that this process can take only $15-20 \mathrm{~min}$, whereas diffusional droplet growth theory without radiation predicted that the duration of the time interval required for droplets to grow up to $100 \mu \mathrm{m}$ in diameter was on the order of hours (Elperin et al. 2015). The absence of a mechanism to explain liquid droplet growth between stable cloud size, roughly $20 \mu \mathrm{m}$ in diameter, where growth by diffusion only (heat conduction and vapor condensation) becomes negligible, and the size where growth by coalescence can take over, $\sim 100-\mu$ m diameter, came to be known as the "condensation-coalescence bottleneck." Different mechanisms to explain the bottleneck have been postulated, such as turbulent mixing, associated supersaturation fluctuations, and radiation-assisted growth. While the former has been well studied (Cooper 1989; Elperin et al. 2015; Li et al. 2019), less attention has been given to radiative enhancement of droplet growth. Guzzi and Rizzi (1980) relaxed 
the prescribed, constant ambient supersaturation assumption of Roach and showed theoretically that longwave cooling allows large droplets to grow while suppressing the growth of smaller droplets. Such differentiating effect on the droplet size spectrum could potentially augment collision-coalescence among droplets. Davies (1985) investigated the assumptions of quasi-steady droplet energy and quasi-steady supersaturation with radiation included and showed that both assumptions are reasonable for typical cloud conditions. Austin et al. (1995) investigated with a one-dimensioned model the combined effect of radiation and collision-coalescence and demonstrated that the time required for the onset of precipitation may be reduced by as much as a factor of 4. Harrington et al. (2000) expanded on the dimensionality and complexity of modeling, and showed that when droplets reside near the top of fogs, stratus or stratocumulus clouds for $12 \mathrm{~min}$ or longer, larger droplets are favored by radiative enhancement and can grow rapidly at the expense of evaporating smaller droplets. They showed that the radiative effect reduces the time required for the onset of drizzle by up to a half hour. Harrington and coworkers (Hartman and Harrington 2005a,b; Marquis and Harrington 2005) continued their modeling investigation by including shortwave (SW) heating into the radiative transfer analysis. The results showed that SW heating partially offsets cloud-top LW cooling, which naturally reduces the influence of LW cooling on droplet growth, but SW heating dominates over LW cooling only at much larger droplet sizes (diameter $\gtrsim 400 \mu \mathrm{m}$ ). As a result of these studies, the effect of radiative cooling, both 1D and 3D (Klinger et al. 2019), by both cloud droplets and water vapor has come to be included in cloud modeling through its effect on raising supersaturation (Bott 2020) but not necessarily in terms of size-dependent droplet radiation (Klinger et al. 2019). A recent theoretical study of size-dependent radiation by water droplets of a range of diameters from $\mu \mathrm{m}$ to mm examined the volumetric or in-depth nature of radiation participation and showed that for LW or infrared radiation, surface absorption/emission could be assumed (Brewster 2015). The implications for radiation on cloud droplet stability in terms of modified Kelvin-Kohler theory were also discussed.

The studies noted above have provided significant theoretical substantiation of the importance of thermal radiation on cloud droplet evolution and the possible importance of radiation in helping to understand and explain the collisioncoalescence bottleneck. Nevertheless, despite these theoretical studies, there are no peer-reviewed publications of experimental work and data obtained in the laboratory that demonstrate and verify radiation-induced droplet growth and that could potentially facilitate assessment of the assumptions and parameterizations used in theoretical studies and numerical simulations of clouddroplet radiative cooling. This absence of laboratory experimental investigation and data and the lack of verification of modeling assumptions are the general motivations for this study.

A related, but more specific, motivation for this study is an ongoing interest in understanding heavy precipitation produced by cloud-top "generating cells" in extratropical, coldseason cyclones. Several studies (e.g., Houze et al. 1981; Plummer et al. 2014) have identified an atmospheric phenomenon involving rapid cooling of cloud droplets at the tops of clouds in extratropical, cold-season cyclones. This rapid cooling happens in cells that have a pronounced vertical structure. These vertical cells have been called "generating cells" because of the role they are believed to play in generating ice "seeder" particles that fall into the lower stratus region of the cloud and become "feeders" for formation of heavy precipitation. It has been suggested (R. M. Rauber and L. Di Girolamo 2019, personal communications) that radiative cooling may play a role in causing temperatures at the tops of these cells to fall low enough (to approximately $-30^{\circ} \mathrm{C}$ ) to induce homogeneous freezing of the droplets and initiate the seeder-feeder process for precipitation formation. The type of influence 3D radiation can have on vertical structure in warm rain clouds has been demonstrated numerically (Klinger et al. 2019). The need to understand this process in freezing clouds is another motivation for the present study.

The objectives of this research were to obtain quantitative measurements of the effect of infrared radiative cooling on cloud-sized water droplet mist evolution experimentally in a laboratory apparatus, both to demonstrate the phenomenon experimentally and to provide data for model comparison and assumption validation, and to compare measured results with predictions based on conventional radiative cooling modeling assumptions. The type of radiative cooling under consideration was that of thermal emission by an assembly of water droplets to a remote, cold radiative sink, similar to what can occur in the atmosphere at the tops of clouds. The laboratory apparatus used a tubular, concentric flow arrangement with saturated air and radiatively participating mist droplets flowing inside a cooled aluminum tube that acted as a radiative sink. Surrounding the core mist flow was a nonradiating annular airflow that acted as a convective/conductive heat shield between the mist and the radiative sink. The behaviors measured in the experiment were mist droplet diameter-distribution change-that is, growth in size by condensation or decrease in size by evaporation-and mist flow temperature change (decrease) as energy radiated from the mist to the heat sink.

This research happened in three phases (Roman 2018; McNichols 2017; Li 2019). Each phase brought about or suggested changes and improvements for the next. In the earlier phases, large droplets appeared in the measurements after radiative cooling which, initially, were thought to be formed by the radiative cooling effect. Upon further investigation it was concluded that some of these large droplets were being formed by other mechanisms. Mitigation of these nonradiative mechanisms was addressed in the subsequent phases of the project. By the end of the third phase the nonradiative mechanisms had mostly been addressed, that is, reduced enough that the effect of radiative cooling could be observed and quantified, at least approximately. The initial phases, the problems encountered and overcome, and preliminary results of the project are described in the appendix, while only the final configuration and results are described below.

\section{Experimental apparatus and methods}

A custom laboratory apparatus was developed that was based on the idea of inducing isobaric radiative cooling of water mist droplets through exposure to a cooled, remote heat 


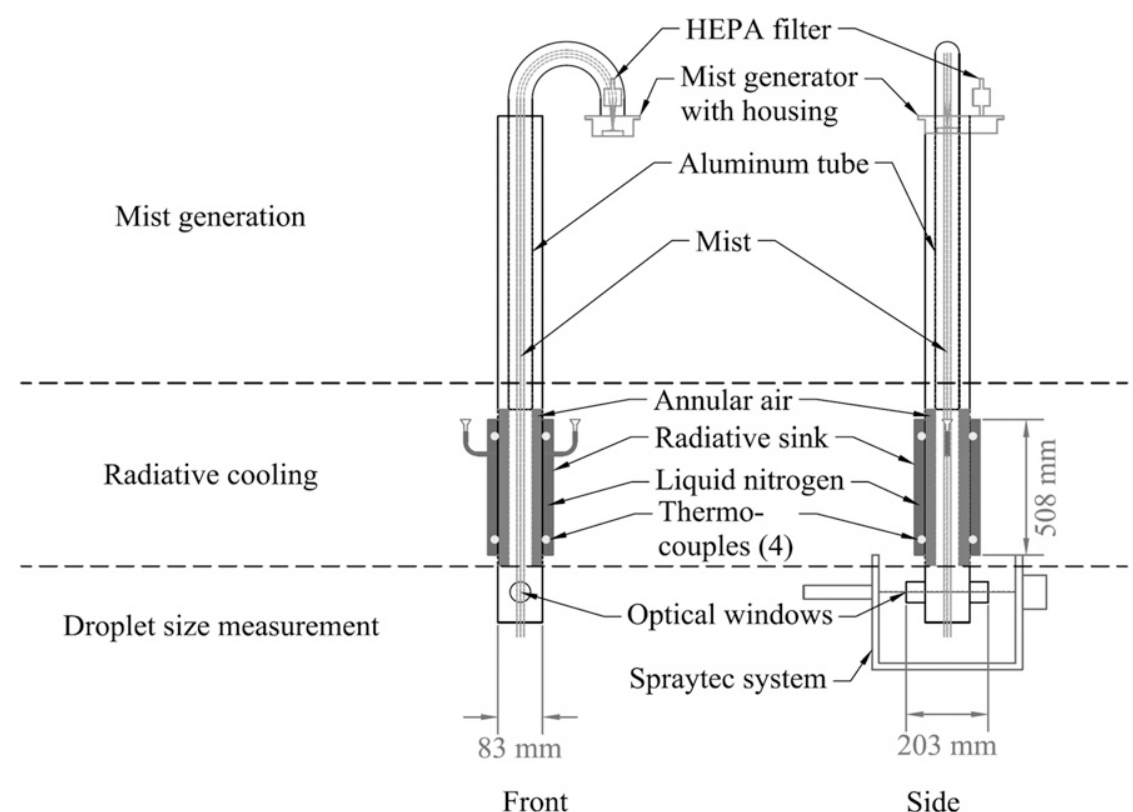

FIG. 1. Experimental apparatus with flow in the top and out the bottom.

sink, while minimizing convective/conductive cooling of the mist. The final version of the apparatus is shown schematically in Fig. 1. The basic component of the apparatus is a vertically oriented $83-\mathrm{mm}$ inside diameter anodized aluminum tube about $1.5 \mathrm{~m}$ in length with mist flow in at the top and out at the bottom. Details of the tube are shown in Fig. 2, with the tube shown horizontally, top (inflow) at the left. A two-phase mixture of saturated air and water droplets, i.e., mist, at $1 \mathrm{~atm}$ flows through the core or center region of the tube. Upstream is a flow-conditioning section with the possibility of inducing convective precooling. Downstream is the main radiative cooling section of length $508 \mathrm{~mm}$. Both sections have provision for cooling the aluminum tube wall by introducing liquid nitrogen into annular regions on the outside of the main aluminum tube. The radiative cooling section has an inner annular airflow region formed by an inner polyethylene (PE) tube $(73 \mathrm{~mm}$ in diameter and $0.1 \mathrm{~mm}$ thick). The $\mathrm{PE}$ tube and annular airflow region were introduced for the purpose of convectively isolating the core mist flow from convective cooling effects of the cooled outer aluminum tube wall by introducing a flow of (nonradiating) air at room temperature in the annulus while allowing radiative cooling of mist droplets via thermal emission to the outer aluminum tube through the infrared-transmissive PE tube. Originally it was believed that the temperature decrease of the mist in the axial flow direction would be very small due to the fact that phase change (primarily condensation of vapor on droplets) would be occurring. Therefore an isothermal PE wall thermal boundary condition was originally sought after. This was done by making the velocity of the annular airflow high enough (Reynolds number $\mathrm{Re} \sim 2000$ ) to prevent the thermal boundary layer from the cooled outer aluminum wall from effectively reaching the inner PE tube before the end (out flow) of the radiative cooling section. It was thought that this (axially isothermal mist) would simplify the modeling process for purposes of comparison with experimental results. As it turned out the assumption of negligible axial mist temperature decrease was eventually found to be too inaccurate and another PE thermal wall condition of being conductively adiabatic was introduced. The latter condition was achieved by reducing the annular airflow velocity from that

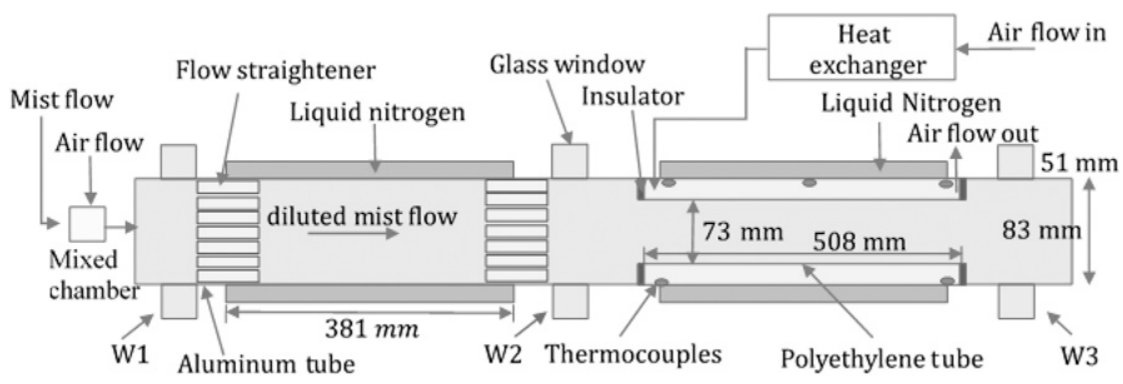

FIG. 2. Detail of flow tube shown horizontally with inflow (top) at left and optical measurement windows (W3) at right. 
of the isothermal wall condition until the annular airflow outlet temperature matched the mist outflow temperature. Type-K thermocouples, placed at the exits of the radiative cooling section and annular airflow section, measured the respective outlet mist flow and annular airflow temperatures. Aluminum tube wall temperature in the radiative sink section was measured by four type-K thermocouples, placed as shown in Fig. 1. Originally both sections of the tube were cooled with liquid nitrogen but after changes were made in the mist-generating system that produced larger droplets, convective precooling became unnecessary and only the radiative section was cooled. Also, originally the tube was oriented horizontally but after possible gravitational settling of droplets in the radial direction became a concern the tube was reoriented vertically.

The inlet mist was generated using piezoelectric transducers, deionized water, and a conditioned laboratory air supply. The transducer eventually selected was an ultrasonic piezoelectric atomizing disc obtained from ICStation (disc diameter of $20 \mathrm{~mm}$ ). This transducer operates at $116 \mathrm{kHz}$, and its disc or plate has tapered holes through it. Droplet diameters produced by the atomizing discs ranged between 0.6 and $20 \mu \mathrm{m}$ with a single mode peak at $7-8 \mu \mathrm{m}$ as measured by a laser diffraction system. This size range was deemed to be suitable for introducing the mist directly into the radiative cooling section without precooling.

The air supply system for the mist was designed so that air supply flow rate could be controlled and measured and the air could be both filtered for particulates and then humidified before having droplets injected. To ensure constant and controlled performance of the droplet atomizing disc, an airtight housing was designed, which also served as a mixing chamber to mix and dilute mist droplets with air. The atomizing disc rested on a sponge soaked with water, which acted as both a support and water supply. Water was filled to the top edge of the sponge before each experiment to prolong the operation time without having to soak the sponge often; in this way the water level could sustain continuous operation of the atomizing disc for four to six hours. Deionized water was used to prevent clogging of the orifices in the atomizing disc and to provide minimal mineral or solute content in the mist droplets. Laboratory air was supplied to the mist generator box, filtered, and then humidified. The filter used was a $0.2 \mu \mathrm{m}$ minicapsule high-efficiency particulate air (HEPA) filter (Gelman model 12122). It is designed to remove naturally occurring aerosol particles larger than about $0.2 \mu \mathrm{m}$ in the supply air from getting into the mixing chamber and being activated. A bubble humidifier using deionized water partially submerged in a warm water bath was built into the air line to humidify the supply air, until condensate formed in the tube at the exit of the humidifier (an indication of the air being saturated). A synthetic fabric mesh was put into the humidifier to increase the contact surface area between the airflow and water. This device helped to prevent low-humidity air from evaporating the droplets. Warm water was acquired from the hot water supply from tap. There was no additional heater to warm the water, but the water in the bath was replaced every 30 min during the experiment to keep the supply air saturated. Airflow rate was regulated and measured through two identical parallel rotameters (Matheson model 11220) with a total capacity of $2.4 \mathrm{~L} \mathrm{~min}^{-1}$.
Droplet diameters were measured at the location shown in Figs. 1 and 2 (W3) both before and after radiative cooling using a laser diffraction system: a Spraytec (Model RTS5008) with Spraytec 97 (RTSizer) software. The system uses a 670-nm wavelength laser and a 450-mm focal-length lens. Laser light scattered (or diffracted) from the incidence direction by droplets near the centerline of the tube passes onto a concentric-ring array detector. The intensity of the scattered light as a function of scattering angle (and therefore detector ring) is droplet-diameter dependent and predictably so by well-known (Mie) scattering theory for homogeneous spheres. Accounting for the optical properties of the droplets, the surrounding medium, and the possibility and degree of multiple scattering between droplets, the software calculates the droplet-diameter distribution in terms of relative volume fraction. For the combination of lens focal length and laser wavelength, the system measures properties over an effective pathlength of $12 \mathrm{~mm}$ (as compared with the tube diameter of $83 \mathrm{~mm}$ ) near the axial centerline of the flow. With the $450-\mathrm{mm}$ focal-length lens, the effective droplet diameter measuring range for the Spraytec system is reportedly $2.5-4000 \mu \mathrm{m}$. Data can still be obtained outside the range but are of reduced accuracy (Malvern Instruments 1999). The error incurred outside this range, say, below $2.5 \mu \mathrm{m}$, was not reported by the system manufacturer.

Experiments were run with both the original isothermal wall condition and the conductively adiabatic wall condition and for various radiant fluxes and various droplet residence times in the radiative cooling section. Radiant flux was varied by varying sink temperature and residence time was varied by varying mistairflow rate. Because of tradeoffs between conflicting experimental conditions, one baseline set of conditions for residence time/flow rate was chosen to focus on. The baseline radiative sink temperature was $-20^{\circ} \mathrm{C}$. A residence time that allowed measurable droplet growth was $80 \mathrm{~s}$, corresponding to a mist flow rate of $1.58 \mathrm{~L} \mathrm{~min}^{-1}$ (airflow rate of $1.50 \mathrm{~L} \mathrm{~min}^{-1}$ ), a radiant cooling section length of $50.8 \mathrm{~cm}$, a flow area of $41.8 \mathrm{~cm}^{2}$, and a mean mist flow velocity of $0.63 \mathrm{~cm} \mathrm{~s}^{-1}$.

For the purpose of estimating droplet residence time in the radiant cooling section, the flow was assumed to be mildly turbulent with an approximately uniform time-average velocity profile across a given radial section, in spite of the relative low time-averaged Reynolds number $(\operatorname{Re} \sim 30)$. This assumption is based on snake camera observations of the flow during operation, which showed that the mist flow exiting the corrugated feed tube and entering into the radiant cooling section had noticeable unsteadiness with swirl and radial velocity component to the motion. While a single-phase flow at this low Re, even with mild entry turbulence, might be expected to stabilize and laminarize as it entered and flowed through the radiant cooling tube, the same cannot be expected of a two-phase flow with active phase change occurring. In this two-phase flow, some droplets (smaller ones) were possibly evaporating, injecting matter into the vapor phase, while others (larger ones) were definitely condensing, removing matter from the vapor phase. This microscopic flow disturbance would have been occurring near virtually every droplet in the flow and is thought to be one of the mechanisms that prevents such two-phase flows from exhibiting the propensity to stabilize via viscous forces that single-phase flows have. (Another mechanism would be gas-droplet relative velocity slip.) 


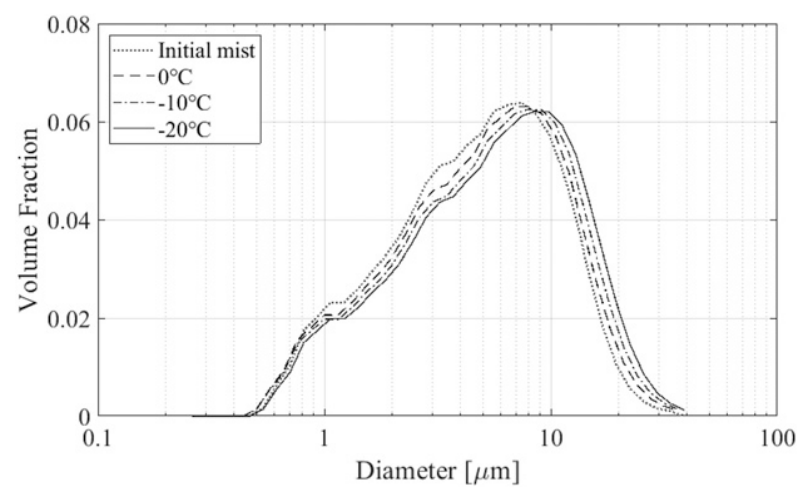

FIG. 3. Smoothed droplet size distributions after $80 \mathrm{~s}$ of radiative cooling with isothermal PE wall condition for $T_{o}=20^{\circ} \mathrm{C}$ and varying $T_{r}$.

\section{Experimental results}

Figure 3 shows the measured volume-fraction size distributions before radiative cooling and after $80 \mathrm{~s}$ of radiative cooling for the isothermal-wall condition for an initial mist temperature of $T_{o}=20^{\circ} \mathrm{C}$ and radiative sink temperatures of $T_{r}=$ $0^{\circ},-10^{\circ}$, and $-20^{\circ} \mathrm{C}$. The distributions shown are the averages of at least five runs. The data for each run have been averaged and then boxcar smoothed using the nearest neighboring points. The maximum coefficients of variation of the measured droplet volume fractions for all runs at the same sink temperature were $0.1 \%, 0.09 \%$, and $0.08 \%$ for the three sink temperatures, $0^{\circ},-10^{\circ}$, and $-20^{\circ} \mathrm{C}$, respectively. Figure 3 shows that the droplet sizes increase after radiative cooling, as evidenced by the shift in the distributions to larger droplet diameters. Furthermore, as $T_{r}$ decreases, that is, as radiative flux increases, the amount of droplet growth is increased.

The mean diameters and liquid droplet concentrations corresponding to Fig. 3 are shown in Table 1. The mean diameters increase with decreasing $T_{r}$, consistent with the shift in distributions shown in Fig. 3. The values of liquid droplet concentration $C_{v}$ shown in Table 1 are the values indicated by the laser diffraction system. Presumably these values are based on a transmission pathlength of $12 \mathrm{~mm}$, which is actually the pathlength of the scattering measurement volume as determined by the optical system, not the transmission signal's pathlength. The actual pathlength for transmission measurement in the experiments is probably longer than $12 \mathrm{~mm}$, but difficult to estimate. Corrected values for droplet volume concentration could be estimated using the linear dependence of volumetric extinction coefficient on droplet volume concentration, assuming a spatially uniform or homogeneous extinction coefficient, and using the linear dependence of optical depth on pathlength. Based on an estimated experimental pathlength of $203 \mathrm{~mm}$ (the distance from window to window in Fig. 2), the corrected values of liquid droplet volume concentration would be smaller than those shown in Table 1 by the ratio of $12 \mathrm{~mm} / 203 \mathrm{~mm}$.

Figure 4 shows the measured volume-fraction size distributions before radiative cooling and after $80 \mathrm{~s}$ of radiative cooling for the conductively adiabatic wall condition for $T_{o}=20^{\circ} \mathrm{C}$ and
TABLE 1 . Indicated liquid water concentrations $C_{v}$, and 43 moment $\left(D_{43}\right)$ of droplet diameter distributions before and after radiative cooling for the same conditions as in Fig. 3.

\begin{tabular}{lrcc}
\hline \hline & \multicolumn{1}{c}{$T_{r}\left({ }^{\circ} \mathrm{C}\right)$} & $C_{v}(\mathrm{ppm})$ & $D_{43}(\mu \mathrm{m})$ \\
\hline Before cooling & - & $60.7 \pm 3.5$ & $6.04 \pm 0.04$ \\
After cooling & $0 \pm 0.5$ & $31.2 \pm 4.3$ & $6.43 \pm 0.05$ \\
& $-10 \pm 0.5$ & $23.2 \pm 2.1$ & $6.82 \pm 0.07$ \\
& $-20 \pm 0.5$ & $25.2 \pm 3.3$ & $7.30 \pm 0.05$ \\
\hline
\end{tabular}

$T_{r}=-20^{\circ} \mathrm{C}$. The discrete data points (unsmoothed) with uncertainty bars represent the average of five runs with corresponding standard deviations. After averaging between runs, boxcar smoothing is done using the nearest neighboring points (smoothed lines). The results in Fig. 4 clearly show that the droplet sizes have increased after $80 \mathrm{~s}$ have elapsed, during which time radiative cooling occurred. Because the nearly conductively adiabatic wall condition has been enforced, the portion of this growth that is due to heat transfer can be attributed primarily to radiation. Figure 4 also includes three modeling curves, which are discussed in section 4 .

The mean diameters, indicated liquid droplet concentrations or total volume fractions $C_{v}$, and mist temperatures corresponding to Fig. 4 are shown in Table 2. The mean $\left(D_{43}\right)$ diameter increased with radiative cooling from 5.5 to $8.4 \mu \mathrm{m}$, consistent with the shift in distributions shown in Fig. 4. The mist temperature decreased by $17^{\circ} \mathrm{C}$ (from $20^{\circ}$ to $3^{\circ} \mathrm{C}$ ) in $80 \mathrm{~s}$ of radiative cooling. This was a significantly bigger temperature drop than would have been expected at the beginning of this study. The estimated net actinic radiative heat flux for the largest (most opaque) droplets would have varied from $180 \mathrm{~W} \mathrm{~m}^{-2}$ at the beginning of the process to $90 \mathrm{~W} \mathrm{~m}^{-2}$ at the end. These numbers correspond to the net blackbody actinic flux based on the droplet (i.e., mist) temperature (at beginning and end of process) and the radiative sink temperature: $\sigma\left(T^{4}-T_{r}^{4}\right)$. They slightly overestimate (by less than $10 \%$ ) the actual actinic fluxes that would be determined if droplet absorption and emission efficiencies (which approach 1 for droplets bigger than $20 \mu \mathrm{m}$ in diameter) were included.

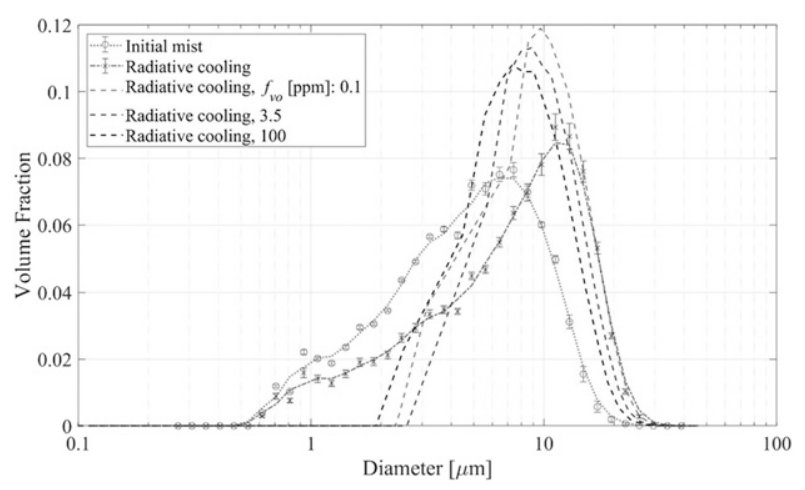

FIG. 4. Measured and predicted $\left(\alpha_{c}=1\right)$ droplet size distributions after $80 \mathrm{~s}$ of radiative cooling with conductively adiabatic PE wall condition for $T_{o}=20^{\circ} \mathrm{C}$ and $T_{r}=-20^{\circ} \mathrm{C}$. 
TABLE 2. Indicated liquid water concentrations $C_{v}$, mist temperature $T$, and 43 moment $\left(D_{43}\right)$ of droplet diameter distributions before and after radiative cooling for the same conditions as in Fig. 4.

\begin{tabular}{lccc}
\hline \hline & $C_{v}(\mathrm{ppm})$ & $T\left({ }^{\circ} \mathrm{C}\right)$ & $D_{43}(\mu \mathrm{m})$ \\
\hline Before cooling & $59.0 \pm 4.4$ & $20.1 \pm 1.4$ & $5.51 \pm 0.04$ \\
After cooling & $67.9 \pm 11.1$ & $2.9 \pm 0.2$ & $8.39 \pm 0.08$ \\
Predicted & & 2.2 & 10.5 \\
\hline
\end{tabular}

\section{Theoretical modeling}

In addition to demonstrating radiation-induced droplet growth experimentally, another objective of this work was to provide data that would facilitate consideration of theoretical modeling assumptions and to compare these measurements with predictions based on conventional radiation-cooling modeling assumptions. In the simplest framework of assumptions, the growth of a pure water droplet of diameter $D_{i}$ in an ambient vapor that is slightly sub- or supersaturated with supersaturation $s$ and temperature $T$, radiating to a remote heat sink at temperature $T_{r}$, can be described by the following combined unsteady droplet mass and quasisteady energy equation (Roach 1976; Barkstrom 1978; Brewster 2015):

$$
\frac{d D_{i}^{2}}{d t}=\frac{s-s_{s}\left(D_{i}\right)+\mathcal{Q}_{r}\left(D_{i}\right) D_{i}}{\left(H+\Psi_{i}\right) / \Gamma_{i}} .
$$

Properties are defined in Table 3 (Brewster 2015). The decrease in temperature induced by droplet radiative heat loss only, (i.e., the outer boundary of the system is conductively

TABLE 3. Parameter definitions and values.

\begin{tabular}{|c|c|c|}
\hline Symbol & Name & Value/formula \\
\hline$a_{H}$ & Parameter in QSS time constant $\sim 1$ & $\sum_{i} D_{v, i}^{\prime}\left(\frac{1+H^{\prime} / \Psi_{i} \mathrm{Le}_{i}}{1+H / \Psi_{i}}\right) \frac{f v_{i}}{D_{i}^{2}} / \sum_{i} D_{v, i}^{\prime} \frac{f v_{i}}{D_{i}^{2}}$ \\
\hline$\alpha_{i}^{\prime}$ & Vapor thermal diffusivity & $k_{i}^{\prime} / \rho C_{p} \mathrm{~m}^{2} \mathrm{~s}^{-1}$ \\
\hline$\alpha_{c, T}$ & Accommodation coefficients: mass, thermal & Variable $(0.04$ or 1$), 1$ \\
\hline$C_{p}, C_{w}$ & Vapor, liquid-droplet specific heats & $1.00,4.18 \mathrm{~kJ} \mathrm{~kg}^{-1} \mathrm{~K}^{-1}$ \\
\hline$D$ or $D_{i}$ & Droplet diameter & $\mathrm{m}$ or $\mu \mathrm{m}$ \\
\hline$D_{v}$ & Binary diffusion coefficient & $1.97 \times 10^{-5}\left([T(\mathrm{~K}) / 256]^{1.685} \mathrm{~m}^{2} \mathrm{~s}^{-1}\right.$ \\
\hline$D_{v, i}^{\prime}$ & $D_{v}$ with kinetic effects & $D_{v} /\left(1+\frac{2 D_{v}}{\alpha_{c} D_{i}} \sqrt{\frac{2 \pi}{R_{v} T}}\right) \mathrm{m}^{2} \mathrm{~s}^{-1}$ \\
\hline$D_{v, m}^{\prime}$ & Mean value of $D_{v, i}^{\prime}$ & $\sum_{i} D_{v, i}^{\prime} D_{i} N v_{i} / \sum_{i} D_{i} N v_{i}=\sum_{i} D_{v, i}^{\prime} D_{i}^{-2} f v_{i} / \sum_{i} D_{i}^{-2} f v_{i}$ \\
\hline$D_{m n}^{m-n}$ & $\begin{array}{l}\text { Moment of droplet diameter distribution ( } m n \text {-moment of } \\
\text { diameter to } m-n \text { power) }\end{array}$ & $\sum_{i}^{l} D_{i}^{m} N v_{i} / \sum_{i} D_{i}^{n} N v_{i}=\sum_{i} D_{i}^{m-3} f v_{i} / \sum_{i} D_{i}^{n-3} f v_{i}$ \\
\hline$D_{K}$ & Kelvin effect (surface tension) parameter & $4 \sigma_{s} /\left(\rho_{\ell} R_{v} T\right) \mathrm{m}$ or $\mu \mathrm{m}$ \\
\hline$f v_{i}$ & Volume fraction of droplets $D_{i}$ & $f v_{o, i}\left(D_{i} / D_{o, i}\right)^{3}\left(T_{o} / T\right)$ \\
\hline$\Gamma_{i}$ & $\begin{array}{l}\text { Droplet evaporation/condensation rate (also conductivity) } \\
\text { parameter }\end{array}$ & $8 k_{i}^{\prime} /\left(\rho_{\ell} h_{\mathrm{fg}}\right)$ \\
\hline$h_{\mathrm{fg}}$ & Water enthalpy of vaporization & $2501-2.34 T\left({ }^{\circ} \mathrm{C}\right) \mathrm{kJ} \mathrm{kg}^{-1}$ \\
\hline$H_{p}, H$ & $\begin{array}{l}\text { Latent heat (or saturation-state temperature sensitivity) } \\
\text { parameters }\end{array}$ & $h_{\mathrm{fg}} /\left(R_{v} T^{2}\right), H_{p}-1 / T$ \\
\hline$k$ & Vapor thermal conductivity & $0.0266[T(\mathrm{~K}) / 293]^{0.808} \mathrm{~W} \mathrm{~m}^{-1} \mathrm{~K}^{-1}$ \\
\hline$k_{i}^{\prime}$ & $k$ with kinetic effects & $k /\left(1+\frac{2 k}{\alpha_{T} D_{i} \rho C_{p}} \sqrt{\frac{2 \pi}{R_{a} T}}\right) \mathrm{W} \mathrm{m}^{-1} \mathrm{~K}^{-1}$ \\
\hline$K_{a, \ell}$ & Liquid water absorption coefficient & $2.33 \times 10^{5} \mathrm{~m}^{-1}$ \\
\hline $\mathrm{Le}_{i}$ & Lewis number & $\alpha_{i}^{\prime} / D_{v, i}^{\prime}$ \\
\hline$P_{s}$ & Water saturation pressure at $T\left({ }^{\circ} \mathrm{C}\right)$ & $\begin{array}{l}0.61138+\left(4.4053 \times 10^{-2}\right) T+\left(1.4594 \times 10^{-3}\right) T^{2}+ \\
\quad\left(2.6092 \times 10^{-5}\right) T^{3}+\left(2.8332 \times 10^{-7}\right) T^{4}+ \\
\quad\left(2.7316 \times 10^{-9}\right) T^{5} \mathrm{kPa}\end{array}$ \\
\hline$\Psi_{i}$ & Diffusive transport parameter & $k_{i}^{\prime} R_{v} T \quad R_{v} C_{p} P$ \\
\hline & & $\overline{D_{v i}^{\prime} h_{\mathrm{fg}} P_{s}}, \overline{R_{a} h_{\mathrm{fg}} P_{s} \mathrm{Le}_{i}}$ \\
\hline$Q_{a}\left(D_{i}\right)$ & Droplet absorption/emission efficiency & $1-2\left\{1-\left[1+\tau\left(D_{i}\right)\right] e^{-\tau\left(D_{i}\right)}\right\} / \tau\left(D_{i}\right)^{2}$ \\
\hline $\mathcal{Q}_{r}\left(D_{i}\right)$ & Droplet radiation parameter & $\frac{Q_{a}\left(D_{i}\right) \sigma\left(T^{4}-T_{r}^{4}\right) H}{2 k_{i}^{\prime}}$ \\
\hline$R_{v}, R_{a}$ & Water-vapor, air gas constants & $0.461,0.287 \mathrm{~kJ} \mathrm{~kg}^{-1} \mathrm{~K}^{-1}$ \\
\hline$\rho$ & Vapor density & $P /\left(R_{a} T\right)=352 / T(\mathrm{~K}) \mathrm{kg} \mathrm{m}^{-3}$ \\
\hline$\rho_{\ell}$ & Liquid water density & $1000 \mathrm{~kg} \mathrm{~m}^{-3}$ \\
\hline & Water-vapor partial density & $(1+s) P_{s} /\left(R_{v} T\right) \mathrm{kg} \mathrm{m}^{-3}$ \\
\hline$s_{s}\left(D_{i}\right)$ & Equilibrium supersaturation at droplet surface (no solute) & $D_{K} / D_{i}$ \\
\hline & Water surface tension & $0.074 \mathrm{~N} \mathrm{~m}^{-1}$ \\
\hline$\tau\left(D_{i}\right)$ & Droplet geometric optical depth & $K_{a, \ell} D_{i}$ \\
\hline
\end{tabular}


TABLE 4. Modeling assumptions.

1) Near-saturated vapor phase $(|s| \ll 1)$

2) Vapor phase dilute in water vapor

3) Dilute droplet assembly (negligible liquid volume fraction, $f v \ll 1$, and mass fraction or ratio, $f v \rho_{\ell} / \rho \ll 1$ )

4) Energy-wise quasi-steady droplets

5) Negligible velocity slip between droplets and vapor

6) Spatially uniform vapor state

7) Thermodynamic equilibrium at droplet surfaces

8) Radiatively nonparticipating vapor phase

9) Effective gray droplet radiative properties based on geometric optics approximations

10) Large, isothermal (blackbody) radiative surroundings

11) Optically thin, nonscattering (in infrared) droplet assembly

12) Negligible solute in droplets

13) Negligible boundary (wall) conductive/convective heat transfer

14) No radiative cooling of droplets before they enter the cooling section or after they leave, and constant (unity) radiative view factor between droplets and radiative heat sink while droplets are in the radiative cooling section (i.e., step function in view factor rather than smoothed)

adiabatic) can be described by the following unsteady vaporphase energy equation, which accounts for the heat conducted to all droplets from the surrounding vapor (Brewster 2015; Li 2019):

$$
\frac{d T}{d t}=12 \sum_{i} \alpha_{i}^{\prime} \frac{f v_{i}}{D_{i}^{2}}\left[\frac{s-s_{s}\left(D_{i}\right)-\mathcal{Q}_{r}\left(D_{i}\right) D_{i} \Psi_{i} / H}{H+\Psi_{i}}\right] .
$$

The change in vapor-phase supersaturation can be obtained by solving the unsteady mass balance on water vapor or unsteady supersaturation (USS) equation,

$$
\begin{aligned}
\frac{1}{(1+s)} \frac{d s}{d t}= & -H_{p} \frac{d T}{d t} \\
& -\frac{3}{2} \frac{\rho_{\ell}}{\rho_{v}} \sum_{i} \frac{\Gamma_{i}}{\left(H+\Psi_{i}\right)} \frac{f v_{i}}{D_{i}^{2}}\left[s-s_{s}\left(D_{i}\right)+\mathcal{Q}_{r}\left(D_{i}\right) D_{i}\right] .
\end{aligned}
$$

The left-hand side term plus the first right-hand side term represent the change in water-vapor mass per unit time of a fixed-mass control volume per unit volume. The second righthand side term is the net rate of water evaporation (or minus the net condensation rate) from all the droplets.

Assumptions associated with these equations are listed in Table 4. The first few assumptions (1-4) are general ones commonly assumed for cloud conditions ( $\mathrm{Li} 2019)$. Assumptions 5-7 are also general ones commonly used in simulations and analyses of cloud radiative cooling, but which are perhaps more questionable than assumptions 1-4. For example, assumption 6 , which allows what might be called the bulk radiative cooling effect to be described, does not account for droplet-scale spatial nonuniformity of supersaturation induced by differential radiative cooling between smaller and larger droplets. To the authors' knowledge, investigation or justification of this assumption has not been reported in the literature. A few assumptions (8-9) are computationally expedient but optional and could be or have been relaxed in detailed modeling studies by invoking spectrally resolved, Mie scattering calculations and radiative transfer, for example, or gas-band radiative properties. The last several assumptions (10-14) are ones specific to the particular laboratory conditions and apparatus of this study. An additional assumption, not in the governing equations, is introduced by the formula for droplet volume fraction $f v_{i}$ shown in Table 3 , which is that the number of droplets of a given diameter $D_{i}$ stays constant throughout the process at whatever it was initially. To the degree that vapor density is constant this would mean that droplet number density also stays constant and thus $f v_{i}$ would vary proportionately with droplet volume. This is origin of the droplet diameterratio cubed factor in Table 3 , which is the primary factor affecting $f v_{i}$ as droplets grow by condensation or shrink by evaporation. Beyond this effect, there is an additional temperature-ratio factor that derives from the vapor (ideal gas) inverse density variation with temperature at constant pressure (and hence inverse droplet number density variation with temperature). This set of assumptions is the simplest set that could reasonably be expected to simulate the radiative cooling process for the laboratory conditions described above, and is representative of conventional radiation-cooling modeling assumptions [apart from the apparatus-specific ones (10-14)]. The governing differential Eqs. (1)-(3) were solved numerically and evaluated with property values shown in Table 3. Before discussing the solutions and comparisons with experimental results, there is one more theoretical point.

Examination of the results from solving Eqs. (1)-(3) numerically indicated that, independent of its initial value, supersaturation reached a quasi-steady value rather quickly, within milliseconds, which value was very closely given by the following relation:

$\sum_{i} \frac{f v_{i}}{D_{i}^{2}}\left[s-s_{s}\left(D_{i}\right)\right]=0 \Rightarrow s=\frac{\sum_{i} \frac{f v_{i}}{D_{i}^{2}}\left[s_{s}\left(D_{i}\right)\right]}{\sum_{i} \frac{f v_{i}}{D_{i}^{2}}}=D_{31}^{2} \sum_{i} \frac{f v_{i}}{D_{i}^{2}}\left[s_{s}\left(D_{i}\right)\right]$.

Perhaps this condition, or something similar, should have been expected, based on Davies's (1985) finding — now widely implemented in cloud modeling (e.g., Klinger et al. 2019; Bott 2020) — of a quasi-steady supersaturation (QSS) condition. 
However, Davies's algebraic expression [see appendix Eq. (A1)] is significantly more complicated than Eq. (4) such that the connection is not immediately obvious. Furthermore, his characteristic time to reach QSS is reported to be on the order of seconds and not milliseconds. These differences, and the fact that a QSS algebraic equation would reduce computational time significantly over the USS differential equation, motivated further theoretical investigation of the QSS condition.

The QSS condition of Eq. (3) can be analyzed as follows. Substituting Eq. (2) into Eq. (3) gives

$$
\begin{aligned}
\frac{1}{(1+s)} \frac{d s}{d t}= & -12 \sum_{i} D_{v, i}^{\prime} \frac{f v_{i}}{D_{i}^{2}}\left\{\left(\frac{1+H_{p} / \Psi_{i} \mathrm{Le}_{i}}{1+H / \Psi_{i}}\right)\left[s-s_{s}\left(D_{i}\right)\right]\right. \\
& \left.-\left(\frac{1-H_{p} / H \mathrm{Le}_{i}}{1+H / \Psi_{i}}\right) \mathcal{Q}_{r}\left(D_{i}\right) D_{i}\right\}
\end{aligned}
$$

Assuming quasi-steady coefficients, that is, if $D_{i}$ and $f v_{i}$ vary over time scales much longer than that with which $s$ equilibrates, Eq. (5) has an exponential solution with a characteristic response time and a quasi-steady solution (as was previously pointed out by Davies (1985):

$$
s=\frac{\sum_{i} D_{v, i}^{\prime} \frac{f v_{i}}{D_{i}^{2}}\left[\left(\frac{1+H_{p} / \Psi_{i} \mathrm{Le}_{i}}{1+H / \Psi_{i}}\right) s_{s}\left(D_{i}\right)+\left(\frac{1-H_{p} / H \mathrm{Le}_{i}}{1+H / \Psi_{i}}\right) \mathcal{Q}_{r}\left(D_{i}\right) D_{i}\right]}{\sum_{i} D_{v, i}^{\prime} \frac{f v_{i}}{D_{i}^{2}}\left(\frac{1+H_{p} / \Psi_{i} \mathrm{Le}_{i}}{1+H / \Psi_{i}}\right)}(\mathrm{QSS})
$$

This QSS expression is equivalent to Eq. (17) of Davies [i.e., our Eq. (A1)] if differences between the analyses noted below are taken into account. However, it is not necessarily obvious how Eq. (6) could be equivalent to Eq. (4), especially when appreciable radiation is present. Yet the numerical results of Eq. (3) are very clear about the applicability of Eq. (4). A key to resolving the apparent differences between Eqs. (4) and (6) can be found in the coefficient of the radiation term in Eq. (6) involving Lewis number. Lewis number (defined here as Le $=$ $\left.D_{v} / \alpha\right)$ for ideal gases is, in the nonkinetic (continuum) regime, independent of pressure and droplet diameter and only slightly dependent on temperature. For the air/water-vapor system it is close to unity, Le $\sim 1.1$. Even for smaller droplets, as kinetic effects (Fukuta and Walter 1970) begin to cause the thermal and species diffusivities to decrease with decreasing droplet diameter (see Table 3), this effect probably applies to both diffusivities at about the same rate such that Le stays of order 1 . The ratio $H_{p} / H$ is the ratio of the two saturation-state temperature sensitivities by the Clausius-Clapeyron relation. The numerator $H_{p}$ is the relative sensitivity of saturation pressure $\left(d \ln P_{s} / d T\right)$; the denominator $H$ is the relative sensitivity of water-vapor saturation partial density $\left(d \ln \rho_{s} / d T\right)$ such that $H_{p} / H=d \ln P_{s} / d \ln \rho_{s}=[1+1 /(H T)] \sim 1.05$ for $H T \sim 20$. Thus the coefficient of the $\mathcal{Q}_{r}\left(D_{i}\right) D_{i}$ term is only a few percent, much smaller than the coefficient $(\sim 1)$ of the $s_{s}\left(D_{i}\right)$ term. Summed over the droplet distribution, the radiation term will usually not be much bigger than the supersaturation term [intense $\mathrm{CO}_{2}$ laser irradiation (Brewster 2015) might be a possible exception but not for atmospheric radiation, even solar irradiation]. Even if the species diffusivity is much smaller than the thermal diffusivity for very small droplets (i.e., $\alpha_{c} \ll 1$ ) such that $\mathrm{Le}_{i} \ll 1$ and thus the coefficient of the radiation sum is of order one or larger, the volume fractions and diameter dependence of the radiation terms compared to the supersaturation terms are such that the radiation term will still be negligible. Furthermore, for comparable species and thermal accommodation coefficients, $\alpha_{c} \sim \alpha_{T} \sim 1$, the parameters in the coefficient of $s_{s}$ involving $\operatorname{Le}_{i}, \Psi_{i}$, and $H$ are relatively independent of droplet diameter and can be cancelled in Eq. (6), leaving only the droplet-diameter dependence of $D_{v, i}^{\prime}$ to differentiate Eq. (6) from Eq. (4). For typical cloud-sized droplets the latter effect is negligible, $D_{v, i}^{\prime} \sim D_{v}$, and thus Eq. (4) is a relatively simple but accurate expression of quasi-steady supersaturation in developed clouds, even under radiative cooling conditions.

The supersaturation equilibration time constant from Eq. (5) is approximately

$$
\tau_{s} \approx \frac{1}{12 \sum_{i} D_{v, i}^{\prime}\left(\frac{1+H^{\prime} / \Psi_{i} \mathrm{Le}_{i}}{1+H / \Psi_{i}}\right) \frac{f v_{i}}{D_{i}^{2}}}=\frac{D_{31}^{2}}{12 a_{H} D_{v, m}^{\prime}}
$$

Like Eq. (4), Eq. (7) is not in an obvious way equivalent to Davies's more complex solution. However, if slight differences between the analyses are taken into account, Davies's expressions can be reduced to Eqs. (5) and (6) and, if in addition the near-unity Lewis-number condition is invoked, they can be reduced further to Eqs. (4) and (7). In his analysis, Davies 1) included vapor-phase radiation (which has been neglected here) as well as droplet radiation and 2) retained the (relatively unimportant) droplet thermal capacitance term with the droplet heating rate set to the ambient air heating rate, which still invokes the QS droplet energy assumption but retains significant algebraic complexity that adds little accuracy to the solution for systems as dilute in droplets as clouds are. He also 3) assumed constant total droplet number density, whereas here droplet number density is allowed to vary according to isobaric ideal-gas density variation. His results for the time constant and quasi-steady solution can be reduced to those corresponding to Eq. (5): 1) if terms involving vaporphase radiation are eliminated [set the fraction of total radiation flux divergence due to the droplet phase in Davies (1985) to unity, $f=1$ ] , 2) if terms involving droplet specific-heat, $C_{w}$ in Davies (1985), are eliminated (droplet thermal capacitance can also be included in $\rho C_{p}$ ), and 3 ) if instead of assuming constant 


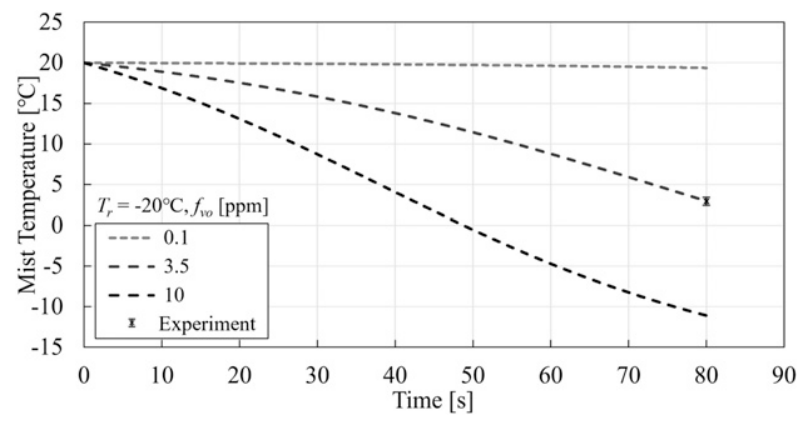

FIG. 5. Predicted $\left(\alpha_{c}=1\right)$ and measured mist temperatures with conductively adiabatic PE wall condition for $T_{o}=20^{\circ} \mathrm{C}$.

total droplet number density (which is equivalent to assuming constant mixture density instead of pressure), mixture density is allowed to vary isobarically and therefore total droplet number density also varies (this has the effect of introducing $H_{p}$, which is replaced by $H$ in Davies's equations). If in addition the Le near-unity condition is invoked, his expressions reduce to the even simpler Eqs. (4) and (7).

One significant point about the present simpler result for quasi-steady supersaturation is that the radiation terms do not appear; only the equilibrium droplet thermodynamic supersaturation terms appear, i.e., Kelvin and Raoult's law (if solute is present) effects. A significant feature of the simpler result for the time constant is that it allows easy verification of Davies's finding that the characteristic time for supersaturation to equilibrate to a perturbation is on the order of seconds for typical cloud conditions. Typical cloud droplet diameters and common moments such as $D_{32}$ and $D_{43}$ are on the order of tens of $\mu \mathrm{m}$ but the 31 moment emphasizes larger diameters such that $D_{31}$ is up to two orders of magnitude larger. Still with $D_{31}$ on the order of millimeters and diffusivities on the order of $10^{-5} \mathrm{~m}^{2} \mathrm{~s}^{-1}$, the time constant by Eq. (7) is on the order of tens of milliseconds, and not seconds, consistent with numerical solution of Eq. (3). Diffusivities on the order of $10^{-5} \mathrm{~m}^{2} \mathrm{~s}^{-1}$ are, of course, for nonkinetic (continuum) conditions, but this order of magnitude also holds generally (i.e., for typical cloud droplet diameter distributions) with an assumed species accommodation (or condensation) coefficient $\alpha_{c}$ of order 1 . To obtain a time constant on the order of seconds would require a much smaller $\alpha_{c}$. There is still lack of agreement about proper values for the accommodation coefficient $\alpha_{c}$; values as low as 0.036 and 0.045 have been reported (Brown and Schowengerdt 1979; Pruppacher and Klett 1997). It appears that values closer to 1 are more generally accepted presently (Seinfeld and Pandis 2006; Langridge et al. 2016), however, and even an assumed value of 0.04 would not quite explain time constants on the order of seconds. The value of diffusion coefficient used by Davies was not reported; however, reference was made to using a much smaller value than that used by Roach. Perhaps a small enough accommodation coefficient would explain why time constants on the order of seconds were obtained. Numerical results from both the USS and QSS models and comparisons with experiments are discussed next.

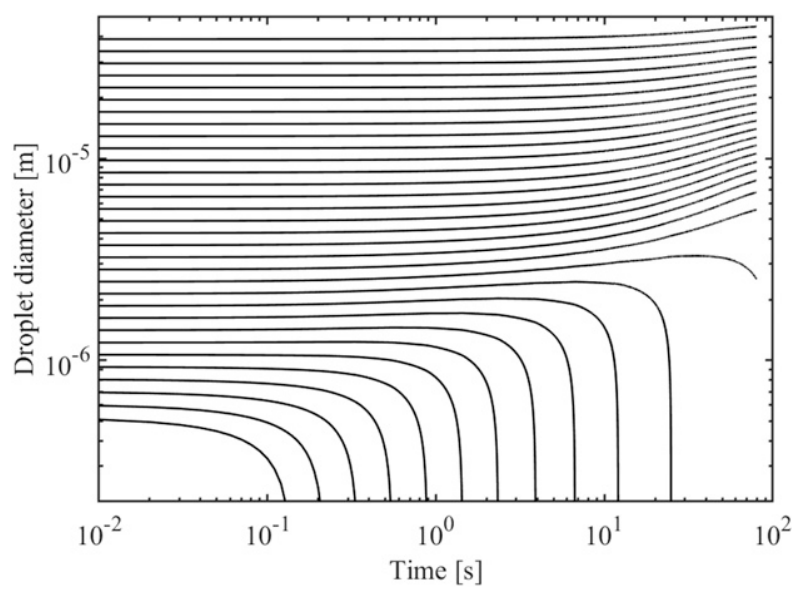

FIG. 6. Predicted $\left(\alpha_{c}=1\right)$ evolution of droplet diameters for conductively adiabatic wall with $T_{o}=20^{\circ} \mathrm{C}, T_{r}=-20^{\circ} \mathrm{C}$, and $f v_{o}=3.5 \mathrm{ppm}$.

\section{Theoretical results and comparison with experiments}

Droplet size distributions and temperatures produced by radiative cooling were predicted using as inputs measured initial size distribution data $\left(f v_{o, i} / f v_{o}\right.$ vs $\left.D_{i}\right)$ shown in Fig. 4 and a range of assumed initial droplet concentration $f v_{o}$ values that were thought to bracket the experimental value. The results shown are for the USS model but they change very little for the QSS model. Figure 5 shows the predicted temperatures for $T_{o}=20^{\circ} \mathrm{C}$ and $T_{r}=-20^{\circ} \mathrm{C}$ (the experimental conditions), and for $f v_{o}=0.1,3.5$, and $10 \mathrm{ppm}$. The value of $3.5 \mathrm{ppm}$ comes from $f v_{o}=59(12 / 203)$, which is based on the indicated $C_{v}=$ $59 \mathrm{ppm}$ and the window-to-window distance of $203 \mathrm{~mm}$ in Fig. 2. Calculations in Figs. 4 and 5 assume $\alpha_{c}=1$ for the accommodation coefficient. Comparing the predicted and measured temperatures in Fig. 5, $f v_{o}=3.5 \mathrm{ppm}$ appears to give the best match of the curves shown: after $80 \mathrm{~s}$ of cooling the predicted temperature is $2.2^{\circ} \mathrm{C}$, only $0.7^{\circ} \mathrm{C}$ below the measured value of $2.9^{\circ} \mathrm{C}$. Reducing the assumed $f v_{o}$ from 3.5 to $0.1 \mathrm{ppm}$ (Fig. 5) increases the predicted temperature to $19^{\circ} \mathrm{C}$; reducing the assumed $f v_{o}$ from 3.5 to $1.0 \mathrm{ppm}$ (not shown in Fig. 5) would increase the predicted temperature to $15^{\circ} \mathrm{C}$; raising $f v_{o}$ to $10 \mathrm{ppm}$ (Fig. 5) decreases the predicted temperature to $-12^{\circ} \mathrm{C}$. Predicted size distributions are shown in Fig. 4 for three assumed values of $f v_{o}=0.1,3.5$, and $100 \mathrm{ppm}(100 \mathrm{ppm}$ was used instead of $10 \mathrm{ppm}$ for better contrast). The size distribution comparison for $3.5 \mathrm{ppm}$ is fair but the predicted peak volume fraction is about $35 \%$ higher than the measured peak and occurs at droplet diameters slightly below $10 \mu \mathrm{m}$, rather than slightly above. Furthermore, droplets below $2.5 \mu \mathrm{m}$ in diameter are (apparently incorrectly) predicted to evaporate completely away after $80 \mathrm{~s}$ of cooling. These discrepancies in the size distributions suggest that one or more of the modeling assumptions enumerated above were not sufficiently satisfied by the experimental conditions, as discussed below.

More details about the case with $f v_{o}=3.5 \mathrm{ppm}$ are shown in Figs. 6 and 7 . Figure 6 shows the calculated droplet diameters as a function of time using the QSS model for $\alpha_{c}=1$. Both USS and QSS models give similar results, leading to the distribution 


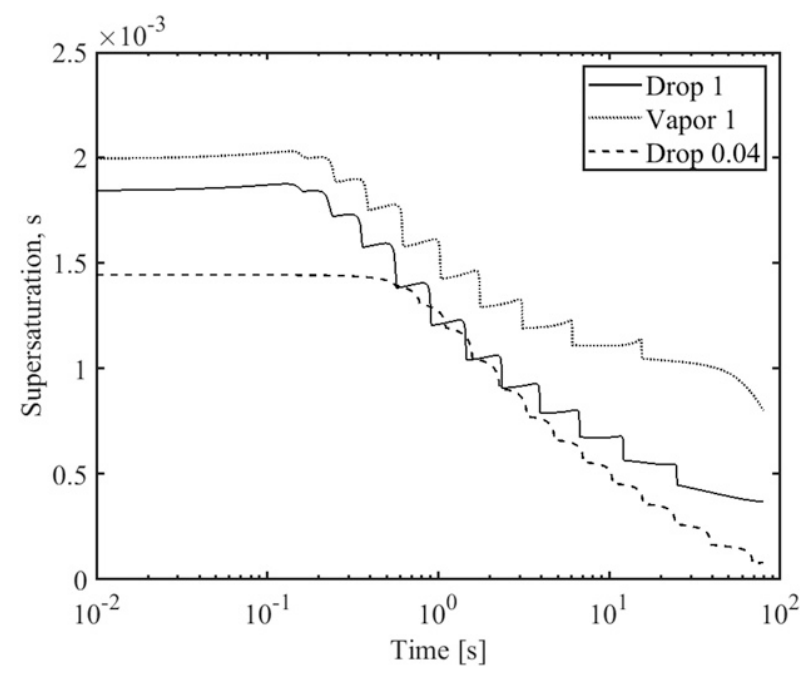

FIG. 7. Predicted evolution of supersaturation for conditions of Figs. 4-6 $\left(f v_{o}=3.5 \mathrm{ppm}\right)$ for droplet-diameter-dependent radiation (Drop) and droplet-diameter-independent radiation (Vapor) with $\alpha_{c}=1$ and 0.04 .

shown in Fig. 4 at $80 \mathrm{~s}$. The droplet diameter distribution is discretized into 32 bins. The smallest bin size (initially $0.536 \mu \mathrm{m}$ ) begins shrinking by evaporation immediately because of a strong Kelvin effect and completely evaporates away at about $0.15 \mathrm{~s}$ as shown in Fig. 6. Computationally, droplets are considered to be completely evaporated and are removed from the droplet phase when their diameter falls below $10 \mathrm{~nm}$. By the time droplets are this small in diameter their bin's volume fraction is so small that they have almost no influence on mixture properties other than supersaturation, so uncertainties in transport properties (i.e., accommodation coefficients), etc., do not influence the calculated results appreciably. While the group of smallest droplets is shrinking in diameter, between 0 and $0.15 \mathrm{~s}$, their equilibrium supersaturation $s_{s}$ increases, causing the ambient supersaturation $s$ to increase slightly as shown in Fig. 7 ("Drop 1" curve). When this smallest group of droplets disappears at $0.15 \mathrm{~s}$, the supersaturation drops suddenly due to these former droplets no longer contributing vapor pressure to the ambient air. At this point, just after $0.15 \mathrm{~s}$, the condition $s<s_{s}\left(D_{i}\right)$ applies to the next larger-size droplet bin (initially $0.616 \mu \mathrm{m}$ ), which evaporates, eventually disappearing at $0.22 \mathrm{~s}$. In this manner the larger-diameter droplets initially grow by condensation, but as the smaller droplets evaporate away, larger ones sequentially begin to evaporate and shrink. After $80 \mathrm{~s}$ of radiative cooling, 11 of the 32 bins have evaporated away. At the time when each group of smaller droplets disappears, supersaturation drops, causing the overall trend of $s$ to decrease as time proceeds and the droplet diameter distribution shifts from smaller to larger diameters with associated smaller Kelvin effect (vapor pressure). The stairstep behavior in Fig. 7 is, of course, an artifact of the droplet diameter bin discretization that would be smoothed away by a continuous distribution representation. This is the computational picture of the process; yet, the disagreement between calculated and measured diameter distributions in Fig. 4 is a

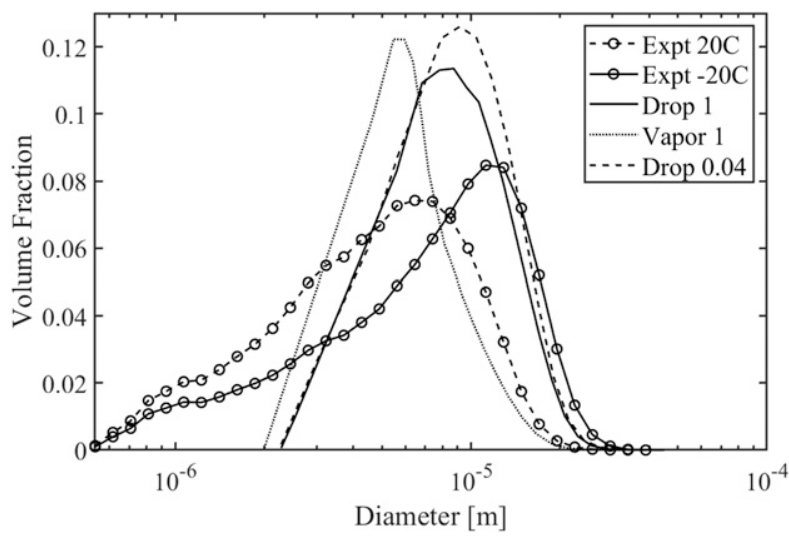

FIG. 8. Predicted and measured (Expt) droplet size distributions for conditions of Figs. 4-6 $\left(f v_{o}=3.5 \mathrm{ppm}\right)$ for droplet-diameterdependent radiation (Drop) and droplet-diameter-independent radiation (Vapor) with $\alpha_{c}=1$ and 0.04 .

reminder that it is probably not the complete or correct picture, that something is still missing from, or incorrect in, the modeling.

Despite the apparent shortcomings in this model with the current, common assumptions, there is value in using it to test the potential influence of other effects, such as the dropletspecific or diameter-specific dependence of radiation and uncertainty in accommodation coefficient. The results are illustrated in Figs. 7 and 8 using the QSS model. First, the dropletspecific or droplet size dependence of radiation was explored. This was done by removing the diameter-dependent term $\mathcal{Q}_{r}\left(D_{i}\right) D_{i}$ that is added in the droplet growth equation, Eq. (1), and adding it to the $s_{s}\left(D_{i}\right)$ term under the sum in the quasisteady supersaturation equation, Eq. (4) or (6). Rather than having diameter-dependent radiation terms, specific to each bin size, as represented by Eq. (1), the effect of those terms is lumped together and put into the QSS supersaturation term. In this way the radiative cooling effect is applied to each droplet equally, regardless of its size (Klinger et al. 2019). This change simulates a given, equivalent radiative flux divergence being emitted/absorbed by the vapor phase rather than by the droplet phase (which change is also made to the vapor energy equation). For net radiative cooling (as opposed to heating), vaporphase radiation would have the effect of increasing supersaturation, $s$, as shown by the modified Eq. (4) or (6). The result of this approach in terms of droplet diameter evolution is shown in Fig. 8 for $\alpha_{c}=1$ in the curve labeled "Vapor 1." The effect is not trivial or negligible. It would appear that the diameter distribution prediction gets worse, that is, shows even less agreement with the measurement ("Expt $-20^{\circ} \mathrm{C}$ ") than the droplet-specific treatment of Eq. (1) ("Drop 1" curve). This type of comparison outcome is to be expected for an experiment in which the radiation was dominated by droplets with negligible vapor radiation contribution. These calculations show that "lumped" or droplet nonspecific radiation modeling, i.e., treating droplet radiation like an equivalent increase in supersaturation in the droplet growth equation, is not an equivalent substitute for droplet-specific radiation. The error 
in applying the "lumped" (droplet nonspecific) radiation approach to droplets in a radiative cooling situation is that smaller droplets, which have smaller absorption/emission efficiencies, get too much radiative cooling effect, retarding their evaporation rate artificially, so that they do not evaporate as quickly as they should, while larger droplets, which have larger absorption/emission efficiencies (approaching unity), get too little radiative cooling effect; thus their condensation rate is artificially low and they do not grow fast enough. The effect of droplet nonspecific radiation on $s$, as noted above, is to increase $s$; the curve for $s$ ("Vapor 1" in Fig. 7) is qualitatively similar to the corresponding droplet-specific curve ("Drop 1") as shown in Fig. 7 but shifted upward. The effect on temperature would be to lower it a few degrees; this can be attributed to the changes in droplet diameter distribution and its effect on radiative properties, which is to enhance the mixture cooling rate. This type of effect of droplet-specific or nonspecific radiation on quasi-steady supersaturation was also calculated by Davies (1985) using a parameter $f$ that shifted the net radiation flux divergence between vapor only $(f=0)$ for complete droplet nonspecific radiation and droplets only $(f=1)$ for complete droplet-specific radiation. His reported trends of $s$ with $f$ and net emitted flux divergence agree, at least qualitatively, with the present results (see discussion of Fig. 9); however, he did not investigate the effect of $f$ on evolution of droplet diameter distribution by doing an unsteady calculation.

The effect of species or mass accommodation coefficient $\alpha_{c}$ was also explored. As noted above, reported values of $\alpha_{c}$ have ranged widely, from an upper bound of 1 to as low as 0.04 . It appears that contamination or impurity plays a role, with the upper value of 1 corresponding to relatively pure water and impurities lowering the value (Mills and Seban 1967; Carey 1992; Mills and Coimbra 2016; Roy et al. 2020). Because deionized water was used in the experiments the baseline value $\alpha_{c}=1$ was used here in modeling as shown by the "Drop 1" curves in Figs. 7 and 8. To explore the effect of $\alpha_{c}$ theoretically its value was decreased from 1 to 0.04 , as shown by the curve labeled "Drop 0.04" in Figs. 7 and 8. The "Drop 0.04" curve appears to shift a little closer to the final experimental curve ("Expt $-20^{\circ} \mathrm{C}$ ") than the baseline "Drop 1" curve $\left(\alpha_{c}=1\right)$. It could be argued perhaps that the $\alpha_{c}=0.04$ curve is a better fit than the baseline calculation, but the match is not good enough to say that this kind of comparison is a way to draw conclusions about $\alpha_{c}$ values. In addition, the value of 0.04 is lower than values presently thought to be acceptable (Langridge et al. 2016), particularly for pure water (Mills and Coimbra 2016). There remains a larger underlying problem in the modeling that needs correcting before this type of comparison will be helpful in making any assessment about accommodation coefficient. The supersaturation time constant by Eq. (7) for the conditions of Figs. 7 and 8 was typically about $20 \mathrm{~ms}$, going only as high as $60 \mathrm{~ms}$ for the case with $\alpha_{c}=0.04$ after $80 \mathrm{~s}$ of cooling.

Figure 9 shows a comparison of various calculated QSS vapor supersaturations according to Eqs. (4), (6), and Davies's (1985) Eq. (17) (see the appendix) for the initial droplet diameter distribution of Fig. 4 (available upon request), $f v=$ $f v_{o}=3.5 \mathrm{ppm}$, and $T=T_{o}=20^{\circ} \mathrm{C}$. To make Fig. 9 be similar to the analogous Fig. 1 in Davies (1985), radiative flux divergence

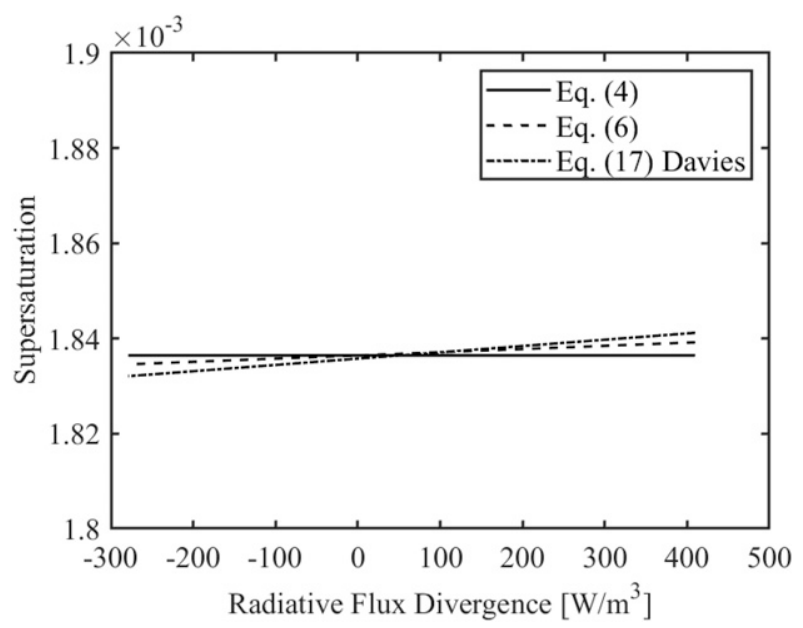

FIG. 9. Variation of quasi-steady vapor supersaturation with droplet-assembly radiative flux divergence according to Eqs. (4), (6), and Davies's (1985) Eq. (17) [our Eq. (A1)] for initial size distribution data of Fig. $4, \alpha_{c}=1, f v=3.5 \mathrm{ppm}, T=20^{\circ} \mathrm{C}$, and $T_{r}$ from $40^{\circ} \mathrm{C}\left(-280 \mathrm{~W} \mathrm{~m}^{-3}\right)$ to $-20^{\circ} \mathrm{C}\left(410 \mathrm{~W} \mathrm{~m}^{-3}\right)$; conditions of Figs. $4-6\left(T_{r}=-20^{\circ} \mathrm{C}\right)$ are far right point $\left(410 \mathrm{~W} \mathrm{~m}^{-3}\right)$.

was varied for fixed droplet diameter distribution and liquid water content. This was achieved in Fig. 9 by varying $T_{r}$ from $-20^{\circ} \mathrm{C}$, the same $T_{r}$ as in Figs. 4-6 (for which divergence ${ }^{1}$ is positive, i.e., net emission of $410 \mathrm{~W} \mathrm{~m}^{-3}$ ), to $40^{\circ} \mathrm{C}$ (for which divergence is negative, $-280 \mathrm{~W} \mathrm{~m}^{-3}$, or net absorption of $280 \mathrm{~W} \mathrm{~m}^{-3}$ ). Over this range of divergences, the value of supersaturation in Fig. 9 is nearly constant at about 0.0018 (the starting value of the solid line in Fig. 7). The main observation from Fig. 9 is how little the radiation terms in Eqs. (6) and (A1) affect QSS supersaturation. Indeed Eq. (4) is a satisfactory representation of QSS vapor supersaturation for radiative cooling of condensing cloud droplets. The slight variations between Davies's Eq. (17) [i.e., Eq. (A1)] and our Eq. (6), both of which include the radiation terms, are probably due to his inclusion of the relatively unimportant droplet thermal capacitance terms $\left[C_{w}\right.$ in Eq. (A1)] and his assumption of conserved droplet number density (and therefore gas density). These small differences do not change the main conclusion from Fig. 9, which is how little the radiation terms affect QSS vapor supersaturation. This conclusion is not quite the same impression as given by the similar plot that is found in Fig. 1 of Davies (1985), and its accompanying discussion. However, there are several differences to keep in mind when comparing these two figures. First, in Fig. 1 of Davies (1985), droplet diameter and volume fraction data come from two cloud models, which have larger droplet diameter distributions (e.g., $\left.D_{43}\right)$ and lower liquid water contents $\left(\rho_{\ell} f_{v}\right)$ than in Fig. 9; second, unknown but probably very low species diffusion coefficients were assumed; third, the assumed radiative flux is also unknown (i.e., effective $T_{r}$ and droplet-to-sink view factor).

\footnotetext{
${ }^{1}$ Radiative divergence is evaluated from $6 \sigma\left(T^{4}-T_{r}^{4}\right) f v \sum Q_{a}\left(D_{i}\right)$ $f v_{i} /\left(f v D_{i}\right)$.
} 
Based on the maximum divergence magnitude in Fig. 1 (Davies 1985), which is $5 \mathrm{~W} \mathrm{~m}^{-3}$, even with the lower liquid water contents, an effective view factor smaller than 1 and/or a temperature difference smaller than $T-T_{r}=20^{\circ} \mathrm{C}-$ $\left(-20^{\circ} \mathrm{C}\right)=40^{\circ} \mathrm{C}$ (or $\left.293-253 \mathrm{~K}=40 \mathrm{~K}\right)$ must have been assumed, such that the maximum droplet net emitted actinic flux must have been smaller than that in Fig. 9 [5.67(2.93 ${ }^{4}-$ $\left.2.53^{4}\right)=185 \mathrm{~W} \mathrm{~m}^{-2}$ ]. Furthermore, Fig. 1 of Davies (1985) includes curves for hypothetical cases in which some or all of the droplet radiation is treated as vapor radiation $(f<1)$. As Davies acknowledges in his summary, droplet radiation really does dominate vapor radiation; therefore, the hypothetical cases for $f=1 / 2$ and 0 , which exaggerate the effect of the radiation terms, can be ignored. Looking at just the $f=1$ case for the upper panel of Fig. 1 in Davies (1985), which has the closest liquid water content $\left(1.03 \mathrm{~g} \mathrm{~m}^{-3}\right)$ to Fig. $9\left(3.5 \mathrm{~g} \mathrm{~m}^{-3}\right)$, it is seen that the supersaturation for divergence (net emission) of $5 \mathrm{~W} \mathrm{~m}^{-3}$ (same as convergence or net absorption of $-5 \mathrm{~W} \mathrm{~m}^{-3}$ ) is about 0.00018 , an order of magnitude smaller than 0.0018 of Fig. 9. [Note the sign change between flux divergence (net emission) in Fig. 9 and flux convergence (net absorption) in Fig. 1 of (Davies (1985).] This order-of-magnitude difference in supersaturation can probably be attributed in part to the larger droplet diameter distribution with modal droplet diameter of $19.4 \mu \mathrm{m}$ in the upper panel of Davies's Fig. 1 versus $D_{43}=5.5 \mu \mathrm{m}$ in Fig. 9 (i.e., lesser Kelvin effect for bigger droplets). The rest of the difference is probably due to unknown differences in accommodation coefficient. The effect of liquid water content comes through the flux divergence as follows. If $\rho_{\ell} f_{v}$ were decreased by an order of magnitude from 3.5 to $0.35 \mathrm{~g} \mathrm{~m}^{-3}$, closer to the cloud model values of Fig. 1 in Davies (1985) (1.03 and $0.14 \mathrm{~g} \mathrm{~m}^{-3}$ ), Fig. 9 would still look the same; the only change would be that the divergence magnitudes on the horizontal axis would also decrease by a factor of 10 , since divergence is proportional to $f v$. Thus, to make Fig. 9 match or look more like Davies's Fig. 1 quantitatively would require increasing the droplet diameters, decreasing the liquid water content, decreasing the maximum value of $\left|T-T_{r}\right|$ below $40 \mathrm{~K}$, and decreasing the accommodation coefficient. The main point of Fig. 9, though, is not in matching Fig. 1 of Davies (1985); it is that, given its simplicity, Eq. (4) is a very good approximation for QSS vapor supersaturation in simulating cloud droplet radiative cooling.

\section{Discussion and conclusions}

One of the results of this study was the discovery of the importance of various unanticipated or unintended phenomena that affected measured droplet size distributions in laboratory experiments. These phenomena included condensation of water vapor on cooled wall surfaces and shedding of droplets therefrom, as well as differential gravitational settling of droplets of varying sizes. Of these phenomena, wall condensation appears to have been solved by eliminating the need for convective precooling and successfully isolating the mist flow from convective cooling in the radiative section. Gravitational settling also appears to have been solved, at least in the radial direction, by vertically orientating the flow and aligning the flow direction with the gravity vector. However, gravitational settling in the axial direction could still play a role even with the vertical flow orientation, for example, in affecting the residence times of droplets of different sizes. It could also potentially introduce another mechanism of droplet size evolution, collision-coalescence, whereby faster moving larger droplets collide and coalesce with slower moving smaller droplets. Estimates of these effects, however, suggest that they were not important in the vertically oriented experiment ( $\mathrm{Li} \mathrm{2019}$; N. Riemer 2019, personal communication).

Another result of this study was the discovery of the importance of the wall thermal boundary condition. Through the evolution of the project, the role and importance of the wall thermal condition and the annular airflow was understood better, which allowed the experiment to be modified to produce conditions more consistent with the desired condition of the mist being convectively/conductively isolated from the remote heat sink and only able to transfer heat by radiation. The original assumption, that the mist flow would remain essentially isothermal during radiative cooling and associated phase change (net condensation) and that an isothermal wall was therefore desirable to minimize convective wall heat transfer, turned out not to be the case.

Another important result of this study was the demonstration of droplet growth during radiative cooling in a laboratory device. The results for both types of wall thermal boundary condition, isothermal and conductively adiabatic, showed that measurable droplet growth occurred during radiative cooling; however, the amount of growth was different depending on the boundary condition. For the same cooling time of $80 \mathrm{~s}$, the same initial temperature of $20^{\circ} \mathrm{C}$, and the same sink temperature of $-20^{\circ} \mathrm{C}$, the conductively adiabatic condition showed more droplet growth $\left(D_{43}\right.$ from 5.5 to $\left.8.4 \mu \mathrm{m}\right)$ than did the isothermal condition $\left(D_{43}\right.$ from 6.0 to $\left.7.3 \mu \mathrm{m}\right)$. This would seem to be consistent with the interpretation that, because the isothermal condition actually transferred heat by conduction/convection into the mist flow, the effect of this heat input was to offset the cooling effect of radiation on droplet growth. But this interpretation is speculative. Further investigation of the isothermal-wall condition and the effect of system-scale heat conduction, both experimentally and by theoretical modeling, should be done.

An important theoretical finding of this study was the derivation of simple algebraic expressions for quasi-steady supersaturation and its time constant. These results were shown to be consistent with the more complex expressions of Davies (1985). However, the time constant here was found to be on the order of milliseconds, not seconds. The difference would appear to be due to assumed species accommodation coefficient. Apparently a very low, but unreported, value of species diffusivity (and thus accommodation coefficient) was assumed by Davies. Perhaps this was a more common assumption at that time (Brown and Schowengerdt 1979). [Another finding Davies attributed to using a very low species diffusivity was refuting Roach's earlier conclusion of droplet growth induced by radiative cooling even under subsaturated conditions. With larger species condensation coefficients, of order approaching 1 (Seinfeld and Pandis 2006; Langridge et al. 2016), Roach's original claim would appear to be exonerated.] The simpler 
expression for QSS supersaturation found here, Eq. (4), is a function of only the droplet size distribution, surface tension and solute parameters, but not radiative transfer. This expression is valid for droplet sizes found in stable clouds, where accommodation coefficient uncertainties do not affect droplet evolution much. The simple expression for QSS time constant found here, Eq. (7), is also valid under the same conditions, and under these conditions is a function of only the continuum binary diffusion coefficient and the $D_{31}$ moment of the droplet size distribution.

That the radiation term in Eq. (5) or (6) (which has a minus sign in front of $H_{p}$ in its coefficient) plays such a minor role relative to the supersaturation term (which has a plus sign in front of $H_{p}$ in its coefficient) is intriguing and begs the question of why, physically, this is so. Mathematically the lineage of signs and coefficients is easy to trace back through the derivation of the equations, but in the process algebraic mixing of terms also mixes interpretations associated with terms, making the physical interpretation more difficult to follow. Nevertheless the difference appears to be related to how supersaturation and its change are dependent on two things: temperature (or energy-transfer quantities) and composition (or mass-transfer quantities), corresponding to the two terms on the right-hand side of Eq. (3). The key difference in signs in front of $H_{p}$ in Eqs. (5) and (6) traces back to the sign difference in front of $\mathcal{Q}_{r}$ in Eqs. (1) and (2). Equation (1) is the unsteady droplet water mass balance. Equation (2) is the unsteady vapor energy balance. The term in large parentheses under the sum on the right-hand side of Eq. (2) represents the quasi-steady droplet energy balance, where each of those terms is the droplet temperature minus the surrounding vapor temperature and could be written as $\left(\Delta T_{i}\right)$. In Eq. (1), increase in droplet mass (positive left-hand side) is aided by condensation of water (positive $s-s_{s}$ term) and by radiative heat away from or out of the droplet (positive $\mathcal{Q}_{r}$ term). Whereas, in the individual terms on the right-hand side of Eq. (2), the quasi-steady droplet energy balance, increase in droplet temperature above vapor temperature (positive $\Delta T_{i}$ ) is aided by condensation of water with its latent heat release (positive $s-s_{s}$ term) and by radiative heat into the droplet (negative $\mathcal{Q}_{r}$ term). This difference in the role of radiation in affecting mass versus energy transfer to the droplet compared with condensation, the associated sign differences in the equations, as well as the similarity of thermal and species diffusivities ( $\mathrm{Le} \sim 1$ ) for air and water vapor seem to be the reasons for the small role radiation plays in determining QSS supersaturation and for the applicability of Eq. (4), even with the occurrence of radiative cooling.

Preliminary comparison of theoretical modeling predictions with laboratory measurements of droplet size and mist temperature provided an opportunity to test modeling assumptions. Simply modifying the quasi-steady supersaturation (i.e., replacing droplet radiation with an equivalent vapor-phase radiation flux divergence, which is equivalent to distributing the radiative flux uniformly over all droplet sizes) was shown not to be an acceptable substitute for including droplet-specific radiation terms in the droplet growth equation. Another common modeling assumption of uncertain validity is assumption 6 of a homogeneous or uniform vapor phase. If, in fact, local spatial variations in supersaturation induced by differential radiative cooling between droplets of different sizes were having an important effect on droplet growth rates, this might help explain the observed discrepancies in droplet size distribution of Fig. 8. Achieving better agreement in size distribution might require relaxing assumption 6 . Nevertheless, the fact that temperature decrease was predicted reasonably well suggests that the bulk radiative cooling effect can be reasonably well represented by a model based on assumption 6 , that is, without accounting for droplet-scale, spatial nonuniformity in supersaturation. Other general assumptions that should be investigated by theoretical analyses are 7 and 9 as well as all apparatus-specific assumptions. The present lack of agreement between modeling and measurements of dropletdiameter distribution suggests that some aspect of the dropletsize-specific behavior in the modeling is incomplete or incorrect with respect to the experiments; it also might be well to repeat the laboratory measurements.

In summary, the results of this study demonstrate the strong effect radiative cooling can have on lowering the temperature of mist in relatively short time scales, i.e., on the order of minutes. This result suggests that radiation might similarly be able to reduce the temperature of pockets of cloud material that have been lifted above the top of towering stratocumulus clouds into an environment where they can radiate effectively to a cold, remote heat sink such as outer space. This finding might have significant implications for cloud microphysics and precipitation formation via "seed and feed" freezing in cloudtop generating cells of extratropical, cold-season cyclones or other storm systems.

Acknowledgments. Support for this work from the U.S. National Science Foundation [Atmospheric and Geosciences (AGS) Division Grant 1457128], the University of Illinois at Urbana-Champaign (UIUC) Mechanical Science and Engineering (MechSE) Department H. G. Soo Professorship (MQB), the UIUC Civil and Environmental Engineering Department Racheff Student Travel Grant (XL), and the UIUC Graduate College Graduate Student Travel Award (XL) is gratefully acknowledged. Helpful discussions with Professors Nicole Riemer, Robert Rauber, and Larry Di Girolamo (UIUC Atmospheric Sciences Department) are also much appreciated, as are suggestions by anonymous reviewers.

\section{APPENDIX}

\section{Initial Phases of Research and Preliminary Results}

Here some description is given of the initial experiments and the problems that were encountered and overcome. Initially the mist was produced using a commercial humidifier or nebulizer. These humidifiers use a piezoelectrically actuated, vibrating plate transducer operating at $1.6 \mathrm{MHz}$. The diameter of these droplets is typically below $10 \mu \mathrm{m}$ with $D_{43}<3 \mu \mathrm{m}$, where $D_{43}$ is the ratio of the fourth to third moment in droplet number density. It was believed that the size of these mist droplets was too small for there to be appreciable radiative cooling or a measurable radiative cooling effect. That is, the droplets were 
thought not to be opaque enough (i.e., not to have high enough absorption/emission efficiency), even in the infrared region, and that droplets bigger than $10-\mu \mathrm{m}$ diameter were required. In addition to that, typical cloud droplets are on the order of $10-\mu \mathrm{m}$ diameter. Therefore, a convective-precooling section was introduced into the apparatus in an effort to induce growth of the droplets before introducing the mist into the radiative cooling section. The convective-precooling section also contained bundles of smaller (3-mm inside diameter) aluminum tubes that acted as flow straighteners. Before the piezoelectrically generated mist was introduced into the flow tube it was diluted by mixing it with an airstream. Figure A1 shows the initial mist droplet size (diameter) distribution generated by the 1.6-MHz surface-wave transducer (Roman 2018). The volume-fraction diameter distribution was bimodal with a larger mode at about 3- $\mu \mathrm{m}$ diameter and a smaller mode at about $0.5-\mu \mathrm{m}$ diameter. The mean diameter was $D_{43}=2.3 \mu \mathrm{m}$.

Figure A1 also shows a typical droplet distribution after the mist flow passed through this original precooling section, which did not use any hydrophobic coating on its surfaces. The conditions for the post-precooling results in Fig. A1 were a mist temperature of $4^{\circ} \mathrm{C}$ and Reynolds number $\mathrm{Re}=53$ (the residence time in main center region of the flow, i.e., not near the walls, of the precooling section was estimated to be $60 \mathrm{~s}$ ). The original bimodal mist diameter distribution (for $D<10 \mu \mathrm{m}$ ) did not change qualitatively upon precooling; the smaller droplets $(D<4 \mu \mathrm{m})$ reduced in volume fraction, presumably due to evaporation, while the larger droplets (for $D>4 \mu \mathrm{m}$ ) increased in volume fraction, presumably due to vapor condensation. The most notable feature of the post-precooling data of Fig. A1 is the appearance of a large droplet mode between 50 and $200 \mu \mathrm{m}$. Initially this new mode was thought to be due to enhanced diffusional condensative growth of droplets flowing near the wall. Convective cooling of the vapor phase increases supersaturation, which augments condensative growth of droplets flowing near the wall. Later (McNichols 2017) it was concluded that vapor was condensing on the wall surfaces in the precooling section, with that condensate being sheared off and broken up into relatively large $(50-200 \mu \mathrm{m})$ droplets. The entrainment of these droplets into the flow was concluded to be the source of the new large droplet mode appearing in Fig. A1. The mean diameter was $D_{43}=29 \mu \mathrm{m}$, though its meaning with such a multimodal distribution is diminished.

Figure A1 also shows a typical droplet size distribution after the mist flow passed through the radiative cooling section with $60 \mathrm{~s}$ of residence time. The wall (radiative sink) temperature was $T_{r}=-28^{\circ} \mathrm{C}$. After radiative cooling, the droplets had a size distribution with an increased mean diameter of $D_{43}=38 \mu \mathrm{m}$. In addition to the general increase in droplet size distribution, the most striking feature of the radiatively cooled distribution in Fig. A1 is the new peak in the large-diameter mode that appears at approximately $70-80 \mu \mathrm{m}$ that is not present in the precooled data. Both the convectively precooled mist and radiatively cooled mist exhibited multimodal droplet size distributions, as shown in Fig. A1. The smaller size regime, below $20 \mu \mathrm{m}$, which was that of the original mist generator droplets, maintained its own, original bimodal character, while the

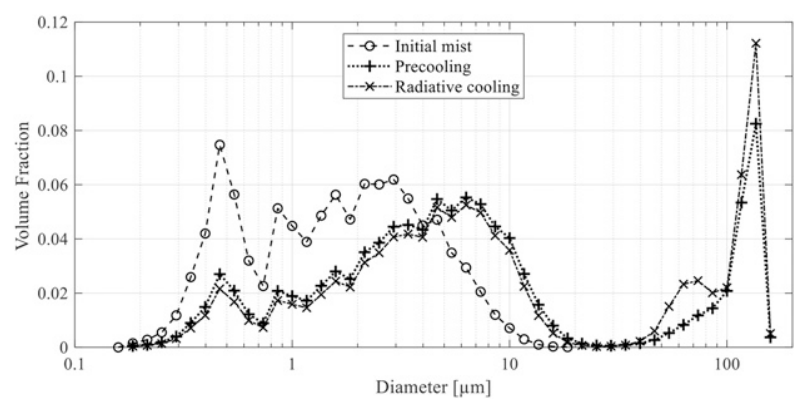

FIG. A1. Droplet size (diameter) distributions from 1.6-MHz mist generator before precooling (initial mist), after convective precooling to $4^{\circ} \mathrm{C}$, and after $60 \mathrm{~s}$ of radiative cooling with $T_{r}=-28^{\circ} \mathrm{C}$.

larger size regime, above $20 \mu \mathrm{m}$, which was essentially monomodal for the convectively cooled mist, became distinctly bimodal for the radiatively cooled mist. Originally the explanation for this new peak was thought to be growth induced by radiative heat loss. Later it was realized that gravitational settling of larger droplets might be responsible for the appearance of the new mode at $70-80 \mu \mathrm{m}$. This realization was the motivation for changes introduced in next phase.

On the basis of the preceding results, changes were introduced into the original steady-flow experimental apparatus. The main change was that a hydrophobic, anticondensation coating was applied to the tube surfaces in the precooling section (McNichols 2017). This change was motivated by the observation of a peak in droplet diameter appearing in the radiatively cooled data at $70-80 \mu \mathrm{m}$ (Fig. A1), and the fact that modeling simulations (Roman 2018) that assumed no gravitational settling could not predict the appearance of a new peak, such as was being observed experimentally. Another change introduced was that the flow straighteners in the precooling section were modified so that the upstream one had good thermal contact with the cooled aluminum tube while the downstream one was thermally insulated from it. It was thought that this would also help in reducing the amount of wall condensation occurring in the downstream flow straightener as periodic reapplication of the coating was required and access to the downstream flow straightener was more limited than was access to the upstream flow straightener. Otherwise the same experimental configuration was used: the horizontal tube orientation and the same $1.6-\mathrm{MHz}$ mist generator were still used.

Figure A2 shows the initial, precooled, and radiatively cooled (after precooling) mist droplet size distributions for an initial mist temperature of $17^{\circ} \mathrm{C}$, a radiative sink temperature of $-28^{\circ} \mathrm{C}$, and a cooling time of $60 \mathrm{~s}$. As expected (since the same transducer was used), the initial distribution is similar to that shown in Fig. A1. The precooled distribution, which can also be compared with Fig. A1, shows that precooling still caused some growth in the smaller droplets $(<10 \mu \mathrm{m})$ generated by the mist generator, but that the use of anticondensation coating on precooling section surfaces appears to eliminate the large droplet mode $(50-200 \mu \mathrm{m})$ of Fig. A1. This seemed to confirm that the large droplet mode 


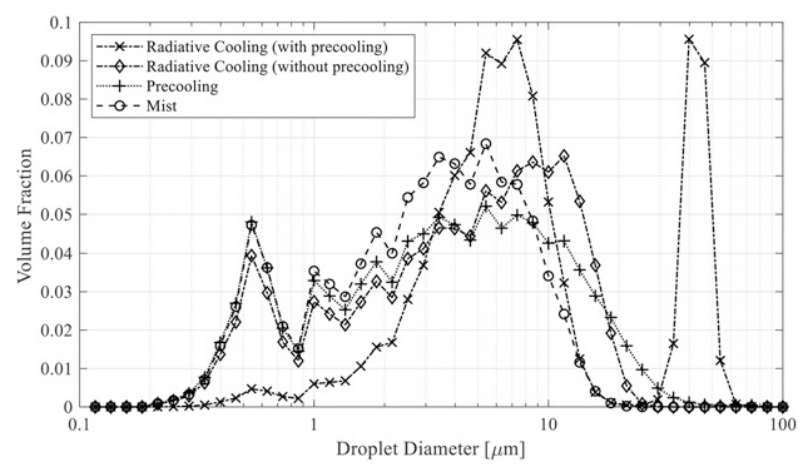

FIG. A2. Droplet size distributions from 1.6-MHz mist generator before precooling (initial mist), after convective precooling with anti-surface-condensation coating, and after $60 \mathrm{~s}$ of radiative cooling $\left(T_{r}=-28^{\circ} \mathrm{C}\right)$, with and without precooling.

was caused by vapor condensation on untreated precooled surfaces and shearing/entrainment of relatively large condensate droplets into the flow in Fig. A1.

The radiatively cooled (after precooling) distribution of Fig. A2 shows the appearance of a new mode of droplets between 30 and $50 \mu \mathrm{m}$ in diameter that was not present in the precooled data. Initially this new mode was thought to be due to radiative cooling of the mist. However, later estimates of gravitational settling time (Li 2019; N. Riemer 2019, personal communication) of various size droplets suggested that the new mode was more likely due to settling of droplets in the radial direction from the top of the tube down into the center of the tube where the measurement windows were located.
The mist flowing near the tube walls probably moved slower than the average or centerline velocity so droplets flowing near the wall (including the top of the tube) would have had time to grow larger than droplets flowing near the centerline. Large droplets formed near the top of the tube conceivably could settle downward into the center of the tube by the time they reached the measurement window and thereby affect the droplet size measurement. This was eventually postulated to be the reason for the 30-50- $\mu \mathrm{m}$ droplet mode that appeared in the radiatively cooled data of Fig. A2, rather than radiative cooling. This postulation was the motivation for additional experimental changes made.

Figure A2 also shows a set of experimental data that was taken without convective precooling. This means the mist flowed through the precooling section but without liquid nitrogen being added. The mist then flowed through the radiative cooling section as usual. The radiatively cooled distribution without precooling does not show the large mode at 30-50 $\mu \mathrm{m}$ but does show some droplet growth in the distribution. The mean $D_{43}$ value grew from 3.6 to $5.5 \mu \mathrm{m}$ upon radiative cooling. This finding seemed to imply that the convective precooling section was part of the problem in producing unwanted large droplets and motivated the search for a new method of generating larger initial mist droplets that would allow the precooling section to be eliminated. With the changes made, as noted above, particularly vertical orientation of the tube, Figs. 3 and 4 show that the larger-mode peaks and multimodal behavior that appeared in Figs. A1 and A2 with horizontal tube orientation were eliminated. Only the single mode or single main peak present in the initial distribution is present in the distributions after radiative cooling.

Here is Eq. (17) of Davies (1985) for QSS supersaturation in the present notation:

$$
\begin{aligned}
& s+1=\left[\frac{12}{h_{\mathrm{fg}} H} \sum_{i} \frac{k_{i}^{\prime} f v_{i} / D_{i}^{2}}{\left\{1+\frac{\Psi_{i} / H}{\left[1+s_{s}\left(D_{i}\right)\right]}\right\}}+\frac{6 \sigma\left(T^{4}-T_{r}^{4}\right)}{h_{\mathrm{fg}}} \sum_{i} \frac{Q_{a}\left(D_{i}\right) f v_{i} / D_{i}}{\left\{1+\frac{\Psi_{i} / H}{\left[1+s_{s}\left(D_{i}\right)\right]}\right\}}\right. \\
& -\frac{6 \sigma\left(T^{4}-T_{r}^{4}\right) \sum_{i} Q_{a}\left(D_{i}\right) f v_{i} / D_{i}}{h_{\mathrm{fg}}\left(\rho_{\ell} f v C_{w}+\rho C_{p}+h_{\mathrm{fg}} H \frac{P_{s}}{R_{v} T}\right)}\left(H \frac{P_{s}}{R_{v} T}+\frac{\rho_{\ell} f v C_{w}}{h_{\mathrm{fg}}} \sum_{i} \frac{f v_{i} / f v}{\left.\left\{1+\frac{\Psi_{i} / H}{\left[1+s_{s}\left(D_{i}\right)\right]}\right\}\right)}\right) / \frac{12}{h_{\mathrm{fg}} H} \sum_{i} \frac{k_{i}^{\prime} f v_{i} / D_{i}^{2}}{\left[1+s_{s}\left(D_{i}\right)+\Psi_{i} / H\right]} .
\end{aligned}
$$

\section{REFERENCES}

Austin, P. H., S. Siems, and Y. Wang, 1995: Constraints on droplet growth in radiatively cooled stratocumulus clouds. J. Geophys. Res., 100, 14 231-14 242, https://doi.org/10.1029/95JD01268.

Barkstrom, B. R., 1978: Some effects of 8-12 $\mu \mathrm{m}$ radiant energy transfer on the mass and heat budgets of cloud droplets. J. Atmos. Sci., 35, 665-673, https://doi.org/10.1175/15200469(1978)035<0665:SEORET > 2.0.CO;2.

Bott, A., 2020: Comparison of a spectral microphysics and a twomoment cloud scheme: Numerical simulations of the cloudtopped marine boundary layer. Bound.-Layer Meteor., 175, 153-178, https://doi.org/10.1007/s10546-020-00501-4.

Brewster, M. Q., 2015: Evaporation and condensation of water mist/cloud droplets with thermal radiation. Int. J. Heat Mass Transfer, $\mathbf{8 8}$, 695-712, https://doi.org/10.1016/j.ijheatmasstransfer.2015.03.055.
Brown, J. T., Jr., and F. D. Schowengerdt, 1979: Water droplet growth measurements in a continuous flow parallel plate thermal diffusion cloud chamber. J. Aerosol Sci., 10, 515-526, https://doi.org/10.1016/0021-8502(79)90007-7.

Carey, V. P., 1992: Liquid-Vapor Phase-Change Phenomena: An Introduction to the Thermophysics of Vaporization and Condensation in Heat Transfer Equipment. Taylor and Francis, $672 \mathrm{pp}$.

Cooper, W. A., 1989: Effects of variable droplet growth histories on droplet size distributions. J. Atmos. Sci., 46, 1301-1311, https:// doi.org/10.1175/1520-0469(1989)046<1301:EOVDGH>2.0.CO;2.

Davies, R., 1985: Response of cloud supersaturation to radiative forcing. J. Atmos. Sci., 42, 2820-2825, https://doi.org/10.1175/ 1520-0469(1985)042<2820:ROCSTR > 2.0.CO;2.

Elperin, T., N. Kleeorin, B. Krasovitov, M. Kulmala, M. Liberman, I. Rogachevskii, and S. Zilitinkevich, 2015: Acceleration of 
raindrop formation due to the tangling-clustering instability in a turbulent stratified atmosphere. Phys. Rev. E, 92, 013012, https://doi.org/10.1103/PhysRevE.92.013012.

Fuchs, N. A., 1959: Evaporation and Droplet Growth in Gaseous Media. Pergamon Press, $72 \mathrm{pp}$.

Fukuta, N., and L. A. Walter, 1970: Kinetics of hydrometeor growth from a vapor-spherical model. J. Atmos. Sci., 27, 1160-1172, https://doi.org/10.1175/1520-0469(1970)027<1160: KOHGFA $>2.0 . \mathrm{CO} ; 2$.

Guzzi, R., and R. Rizzi, 1980: The effect of radiative exchange on the growth of a population of droplets. Contrib. Atmos. Phys., 53, 351-365.

Harrington, J. Y., G. Feingold, and W. R. Cotton, 2000: Radiative impacts on the growth of a population of drops within simulated summertime Arctic stratus. J. Atmos. Sci., 57, 766-785, https://doi.org/10.1175/1520-0469(2000)057<0766: RIOTGO $>2.0 . \mathrm{CO} ; 2$.

Hartman, C. M., and J. Y. Harrington, 2005a: Radiative impacts on the growth of drops within simulated marine stratocumulus. Part I: Maximum solar heating. J. Atmos. Sci., 62, 2323-2338, https://doi.org/10.1175/JAS3477.1.

$\longrightarrow$, and — 2005b: Radiative impacts on the growth of drops within simulated marine stratocumulus. Part II: Solar zenith angle variations. J. Atmos. Sci., 62, 2339-2351, https://doi.org/ 10.1175/JAS3478.1.

Houze, R. A., Jr., S. A. Rutledge, T. J. Matejka, and P. V. Hobbs, 1981: The mesoscale and microscale structure and organization of clouds and precipitation in midlatitude cyclones. III: Air motions and precipitation growth in a warm frontal rainband. J. Atmos. Sci., 38, 639-649, https://doi.org/10.1175/15200469(1981)038<0639:TMAMSA > 2.0.CO;2.

Klinger, C., G. Feingold, and T. Yamaguchi, 2019: Cloud droplet growth in shallow cumulus clouds considering 1-D and 3-D thermal radiative effects. Atmos. Chem. Phys., 19, 6295-6313, https://doi.org/10.5194/acp-19-6295-2019.

Langridge, J. M., M. S. Richardson, D. A. Lack, and D. M. Murphy, 2016: Experimental evidence supporting the insensitivity of cloud droplet formation to the mass accommodation coefficient for condensation of water vapor to liquid water. Geophys. Res. Lett., 43, 6650-6656, https://doi.org/10.1002/ 2016 GL069328.

Li, X., 2019: Radiation-induced condensational growth of cloud-sized mist droplets. M.S. thesis, Dept. of Civil and
Environmental Engineering, University of Illinois at UrbanaChampaign, $99 \mathrm{pp}$.

Li, X.-Y., G. Svensson, A. Brandenburg, and N. E. Haugen, 2019: Cloud-droplet growth due to supersaturation fluctuations in stratiform clouds. Atmos. Chem. Phys., 19, 639-648, https:// doi.org/10.5194/acp-19-639-2019.

Malvern Instruments, 1999: Spraytec installation manual. Malvern Instruments Doc. MAN0300, $50 \mathrm{pp}$.

Marquis, J., and J. Y. Harrington, 2005: Radiative influences on drop and cloud condensation nuclei equilibrium in stratocumulus. J. Geophys. Res., 110, D10205, https://doi.org/10.1029/ 2004JD005401.

McNichols, E. O., 2017: Water droplet growth enhancement through thermal radiation. M.S. thesis, Dept. of Mechanical Science and Engineering, University of Illinois at UrbanaChampaign, 65 pp.

Mills, A. F., and R. A. Seban, 1967: The condensation coefficient of water. Int. J. Heat Mass Transfer, 10, 1815-1827, https:// doi.org/10.1016/0017-9310(67)90052-X.

— , and C. F. M. Coimbra, 2016: Heat Transfer. Temporal, 978 pp.

Plummer, D. M., G. M. McFarquhar, R. M. Rauber, and B. F. Jewett, 2014: Structure and statistical analysis of the microphysical properties of generating cells in the comma head region of continental winter cyclones. J. Atmos. Sci., 71, 41814203, https://doi.org/10.1175/JAS-D-14-0100.1.

Pruppacher, H. R., and J. D. Klett, 1997: Microphysics of Clouds and Precipitation. Kluwer, $954 \mathrm{pp}$.

Reynolds, O., 1877: On the manner in which raindrops and hailstones are formed. Mem. Proc. Manchester Lit. Philos. Soc., 3, 48-60.

Roach, W. T., 1976: On the effect of radiative exchange on the growth by condensation of a cloud or fog droplet. Quart. J. Roy. Meteor. Soc., 102, 361-372, https://doi.org/10.1002/qj.49710243207.

Roman, K. K., 2018: Radiative impacts on water mist/cloud droplet condensative growth. Ph.D. dissertation, University of Illinois at Urbana-Champaign, $141 \mathrm{pp}$.

Roy, S., M. E. Diveky, and R. Signorell, 2020: Mass accommodation coefficients of water on organics from complementary photoacoustic and light scattering measurements on lasertrapped droplets. J. Phys. Chem. C, 124, 2481-2489, https:// doi.org/10.1021/acs.jpcc.9b09934.

Seinfeld, J. H., and S. N. Pandis, 2006: Atmospheric Chemistry and Physics. John Wiley and Sons, 1203 pp. 